\title{
Zeta Functions of Monomial Deformations of Delsarte Hypersurfaces
}

\author{
Remke KLOOSTERMAN
}

Università degli Studi di Padova, Dipartimento di Matematica, Via Trieste 63, 35121 Padova, Italy

E-mail: klooster@math.unipd.it

URL: http://www.math.unipd.it/ klooster/

Received June 09, 2017, in final form November 01, 2017; Published online November 07, 2017 https://doi.org/10.3842/SIGMA.2017.087

\begin{abstract}
Let $X_{\lambda}$ and $X_{\lambda}^{\prime}$ be monomial deformations of two Delsarte hypersurfaces in weighted projective spaces. In this paper we give a sufficient condition so that their zeta functions have a common factor. This generalises results by Doran, Kelly, Salerno, Sperber, Voight and Whitcher [arXiv:1612.09249], where they showed this for a particular monomial deformation of a Calabi-Yau invertible polynomial. It turns out that our factor can be of higher degree than the factor found in [arXiv:1612.09249].
\end{abstract}

Key words: monomial deformation of Delsarte surfaces; zeta functions

2010 Mathematics Subject Classification: 14G10; 11G25; 14C22; 14J28; 14J70; 14Q10

\section{Introduction}

Fix a finite field $\mathbf{F}_{q}$ and a positive integer $n$. In this paper we study a particular class of deformations of Delsarte hypersurfaces in $\mathbf{P}_{\mathbf{F}_{q}}^{n}$. There has been an extensive study of the behaviour of the zeta function in families of varieties. First results were obtained by Dwork (e.g., [9]) and Katz [12]. In the latter paper the author studies a pencil of hypersurface in $\mathbf{P}^{n}$ and describe a differential equation, whose solution is the Frobenius matrix on the middle cohomology for a general member of this pencil.

More recently, the behaviour of the zeta function acquired renewed interest because of two interesting (and very different) applications. Candelas, de la Ossa and Rodriguez-Villegas [6] studied the behaviour of the zeta family in a particular family of quintic threefolds in $\mathbf{P}^{4}$, with a particular interest in phenomena, analogous to phenomena occurring in characteristic zero related with mirror symmetry and let to many subsequent papers by various authors. Another application of Katz' differential equation can be found in algorithms to determine the zeta function of a hypersurface efficiently (see $[17,18]$ ).

The main aim of this paper is to generalize and to comment on a recent result of Doran, Kelly, Salerno, Sperber, Voight and Whitcher [8] on the zeta function of certain pencils of Calabi-Yau hypersurfaces. For a more extensive discussion on the history of this particular result we refer to the introduction of [8].

To describe the main results from [8], fix a matrix $A:=\left(a_{i, j}\right)_{0 \leq i, j \leq n}$ with nonnegative integral coefficients and nonzero determinant. Then with $A$ we can associate the polynomial

$$
F_{A}:=\sum_{i=0}^{n} \prod_{j=0}^{n} x_{i}^{a_{i, j}} .
$$

This paper is a contribution to the Special Issue on Modular Forms and String Theory in honor of Noriko Yui. The full collection is available at http://www.emis.de/journals/SIGMA/modular-forms.html 
Assume that the entries of $A^{-1}(1, \ldots, 1)^{\mathrm{T}}$ are all positive, say $\frac{1}{e}\left(w_{0}, \ldots, w_{n}\right)$ with $e, w_{i} \in \mathbf{Z}_{>0}$. Then $F_{A}$ defines a hypersurface of degree $e$ in $\mathbf{P}_{\mathbf{F}_{q}}\left(w_{0}, \ldots, w_{n}\right)$. Assume that we choose $A$ such that $\operatorname{gcd}(q, e)=1$. (Equivalently, we may assume that $\operatorname{gcd}(\operatorname{det}(A), q)=1$.)

If the hypersurface is geometrically irreducible then we call it a Delsarte hypersurface. A subvariety $X \subset \mathbf{P}\left(w_{0}, \ldots, w_{n}\right)$ is called quasismooth if the affine quasicone of $X$ is smooth away from the vertex. If $F_{A}$ defines a quasismooth hypersurface then $F_{A}$ is called an invertible polynomial. If $F_{A}=0$ defines a Calabi-Yau manifold, i.e., $e=n+1$, then we can consider the one-parameter family $X_{A, \psi}$ given by the vanishing of

$$
F_{A}-(n+1) \psi \prod_{i=0}^{n} x_{i}
$$

The factor $-(n+1)$ is included for historic reasons. In the sequel we will work with the parameter $\lambda=-(n+1) \psi$ for simplicity.

In a recent preprint Doran, Kelly, Salerno, Sperber, Voight and Whitcher [8] showed the following result (using Dwork cohomology and some results on the Picard-Fuchs equation):

Theorem $1.1([8])$. Let $A$ and $A^{\prime}$ be $(n+1) \times(n+1)$-matrices with nonnegative entries such that $F_{A}$ and $F_{A^{\prime}}$ are invertible Calabi-Yau polynomials of degree $n+1$. Assume that $(1, \ldots, 1)^{\mathrm{T}}$ is an eigenvector of both $A$ and $A^{\prime}$ and that

$$
\operatorname{gcd}\left(q,(n+1) \operatorname{det}(A) \operatorname{det}\left(A^{\prime}\right)\right)=1 \text {. }
$$

Moreover, assume that $(1, \ldots, 1) A^{-1}$ and $(1, \ldots, 1) A^{-1}$ are proportional. Then for any $\psi \in \mathbf{F}_{q}$ such that $X_{A, \psi}$ and $X_{A^{\prime}, \psi}$ are smooth and nondegenerate we have that the polynomials

$$
\left(Z\left(X_{A, \psi}, T\right) \prod_{i=0}^{n-1}\left(1-q^{i} T\right)\right)^{(-1)^{n}} \quad \text { and } \quad\left(Z\left(X_{A^{\prime}, \psi}, T\right) \prod_{i=0}^{n-1}\left(1-q^{i} T\right)\right)^{(-1)^{n}}
$$

have a common factor of degree at least the order of the Picard-Fuchs equation of $X_{A, \psi}$.

For a precise definition of nondegenerate we refer to the paper [8]. The condition $(1,1, \ldots, 1)^{\mathrm{T}}$ is an eigenvector of $A$ implies that $X_{A, \psi} \subset \mathbf{P}^{n}$. The condition $(1, \ldots, 1) A^{-1}$ is proportional to $(1, \ldots, 1) A^{-1}$ is the same as the condition dual weights being equal from the paper [8], whenever the latter condition is defined.

In this paper we prove a generalisation of this result. We aim to allow more matrices $A$, more vectors a, to drop the Calabi-Yau assumption, to have a simpler nondegenerate assumption and to find a common factor of higher degree. Moreover, as a by-prodcut of our approach we obtain additional information on the degree of the factor found in [8].

To be more precise, we start again with an invertible $(n+1) \times(n+1)$-matrix $A$ such that $X_{A, 0}$ is quasismooth, but we drop the Calabi-Yau condition. Let $d$ be an integer such that $B:=d A^{-1}$ has integral entries. Let $\mathbf{w}=\left(w_{0}, \ldots, w_{n}\right):=B(1, \ldots, 1)^{\mathrm{T}}$. If all the $w_{i}$ are positive then $F_{A}$ defines a hypersurface in the weighted projective space $\mathbf{P}(\mathbf{w})$.

Fix now a vector $\mathbf{a}:=\left(a_{0}, \ldots, a_{n}\right)$ such that $a_{i} \in \mathbf{Z}_{>0}$, the entries of $\mathbf{b}:=\mathbf{a} B$ are nonnegative and $\sum_{i=0}^{n} a_{i} w_{i}=d$. Then $F_{A, \psi}:=F_{A}-(n+1) \psi \prod_{i=0}^{n} x_{i}^{a_{i}}$ defines a family of hypersurfaces $X_{\psi}$ in $\mathbf{P}(\mathbf{w})$ each birational to a quotient of $Y_{\psi} \subset \mathbf{P}^{n}$ given by

$$
\sum_{i=0}^{n} y_{i}^{d}-(n+1) \psi \prod_{i=0}^{n} y_{i}^{b_{i}}
$$


This is a one-dimensional monomial deformation of a Fermat hypersurface. It is easy to determine for which values of $\psi$ the hypersurface is smooth [15, Lemma 3.7]. The idea to study Delsarte hypersurface by using their Fermat cover dates back to Shioda [20] and has then been used by many authors for to discuss solve problems concerning Delsarte hypersurfaces by considering a similar problem on Fermat surfaces. Recent applications of this idea, in contexts similar to our setup, can be found in $[4,5,13]$.

Take now a further $(n+1) \times(n+1)$ matrix $A^{\prime}$ and a vector $\mathbf{a}^{\prime}$ yielding a second family $X_{\psi}^{\prime}$ in a possibly different weighted projective space.

It is straightforward to show that if $\mathbf{a} A^{-1}$ and $\mathbf{a}^{\prime}\left(A^{\prime}\right)^{-1}$ are proportional then the families $X_{\psi}, X_{\psi}^{\prime}$ have a common cover of the type $Y_{\psi}$, i.e., there exist subgroup schemes $G$ and $G^{\prime}$ of the scheme of automorphisms $\operatorname{Aut}\left(Y_{\psi}\right)$ such that $G$ and $G^{\prime}$ are defined over $\mathbf{F}_{q}, Y_{\psi} / G$ is birational to $X_{\psi}$ and $Y_{\psi} / G^{\prime}$ is birational to $X_{\psi}^{\prime}$. The automorphisms in $G$ and $G^{\prime}$ are so-called torus or diagonal automorphisms, i.e., each automorphism multiplies a coordinate with a root of unity. In particular, $G$ and $G^{\prime}$ are finite abelian groups. We will use this observation to show that:

Theorem 1.2. Let $A$ and $A^{\prime}$ be $(n+1) \times(n+1)$-matrices with nonnegative entries, such that the entries of $\left(w_{0}, \ldots, w_{n}\right)^{\mathrm{T}}:=A^{-1}(1, \ldots, 1)^{\mathrm{T}}$ and of $\left(w_{0}^{\prime}, \ldots, w_{n}^{\prime}\right)^{\mathrm{T}}:=A^{\prime-1}(1, \ldots, 1)^{\mathrm{T}}$ are all positive and $\operatorname{gcd}\left(q, \operatorname{det}(A) \operatorname{det}\left(A^{\prime}\right)\right)=1$. Fix two vectors $\mathbf{a}:=\left(a_{0}, \ldots, a_{n}\right)$ and $\mathbf{a}^{\prime}:=\left(a_{0}^{\prime}, \ldots, a_{n}^{\prime}\right)$ consisting of nonnegative integers such that the equalities $\sum_{i=0}^{n} a_{i} w_{i}=1$ and $\sum_{i=0}^{n} a_{i}^{\prime} w_{i}^{\prime}=1$ hold and such that $\mathbf{a} A$ and $\mathbf{a}^{\prime} A^{\prime}$ are proportional. Let $X_{\psi}, X_{\psi}^{\prime}, Y_{\psi}, G$ and $G^{\prime}$ as above. Denote with $G . G^{\prime}$ the subgroup of $\operatorname{Aut}\left(Y_{\psi}\right)$ generated by $G$ and $G^{\prime}$.

1. If $Y_{\psi}$ is smooth then the characteristic polynomial of Frobenius acting on $H^{n-1}\left(Y_{\psi}\right)^{G . G^{\prime}}$ divides both the characteristic polynomial of Frobenius acting on $H^{n-1}\left(X_{\psi}\right)$ and the characteristic polynomial of Frobenius on $H^{n-1}\left(X_{\psi}^{\prime}\right)$.

2. If, moreover, $(1, \ldots, 1)$ is an eigenvector of both $A$ and $A^{\prime}$ and both $X_{\psi}$ and $X_{\psi}^{\prime}$ are smooth then we have that the polynomials

$$
\left(Z\left(X_{\psi}, T\right) \prod_{i=0}^{n-1}\left(1-q^{i} T\right)\right)^{(-1)^{n}} \quad \text { and } \quad\left(Z\left(X_{\psi}^{\prime}, T\right) \prod_{i=0}^{n-1}\left(1-q^{i} T\right)\right)^{(-1)^{n}}
$$

have a common factor of positive degree.

In the second section we will prove this result under slightly weaker, but more technical hypothesis, see Theorem 2.18 and Corollary 2.21. Moreover, in Proposition 2.24 we will show that the factor constructed in the proof of Theorem 1.1 divides the characteristic polynomial of Frobenius acting on $H^{n-1}\left(Y_{\psi}\right)^{G \cdot G^{\prime}}$. We will give examples where our factor has higher degree.

Note that the quotient map $Y_{\psi} \rightarrow X_{\psi}$ is a rational map. If it were a morphism then it is straightforward to show that the characteristic polynomial of $H^{n-1}\left(Y_{\psi}\right)^{\text {G.G }} G^{\prime}$ divides the characteristic polynomial of Frobenius on $H^{n-1}\left(X_{\psi}\right)$. Hence, large part of the proof is dedicated to show that passing to the open where the rational map is a morphism does not kill any part of $H^{n-1}\left(Y_{\psi}\right)^{G \cdot G^{\prime}}$.

In the course of the proof of Theorem 1.2 we show that we can decompose $H^{n-1}\left(X_{\psi}\right)$ as a direct sum of two Frobenius stable subspaces, namely

$$
H^{n-1}\left(X_{\psi}\right)=H^{n-1}\left(Y_{\psi}\right)^{G} \oplus C .
$$

Similarly, we show that can decompose $H^{n-1}\left(Y_{\psi}\right)^{G}=H^{n-1}\left(Y_{\psi}\right)^{G_{\max }} \oplus W_{\psi}$, where $W_{\psi}$ is Frobenius stable, and $G_{\max }$ is the maximal group of torus automorphisms acting on the family $Y_{\psi}$. 
The appearance of $C$ is related with the fact that the quotient map is only a rational map rather than a morphism. For most choices of $\left(a_{0}, \ldots, a_{n}\right)$ we have that $C$ is independent of $\psi$ and in that case we can express $C$ in terms of the cohomology of cones over Fermat hypersurfaces. Hence the Frobenius action on $C$ is easy to determine. To calculate the Frobenius action on the complementary subspace $H^{n-1}\left(Y_{\psi}\right)^{G}$ we can use the methods from [15] to express the zeta function in terms of generalised $p$-adic hypergeometric functions.

This brings us to another observation from [8]: In [8, Section 5] the authors consider five families of quartic $K 3$ surfaces which have a single common factor of the zeta function of degree 3 . They show that every other zero of the characteristic polynomial of Frobenius on $H^{2}$ is of the form $q$ times a root of unity. Assuming the Tate conjecture for $K 3$ surfaces (which is proven for most $K 3$ surfaces anyway) we deduce that the (geometric) Picard number is at least 19.

This result is a special case of the following phenomena: if for a lift to characteristic zero $h^{n-1,0}\left(H^{n}\left(Y_{\psi}\right)^{G_{\max }}\right)=h^{n-1,0}\left(H^{n-1}\left(X_{\psi}\right)\right)$ holds then it turns out that both $W_{\psi}$ and $C$ are Tate twists of Hodge structures of lower weight. In the $K 3$ case, $W_{\psi}$ and $C$ are Hodge structures of pure (1,1)-type. By the Lefschetz' theorem on (1,1)-classes, they are generated by classes of divisors. In particular, for each of the five families the lifts to characteristic zero have Picard number at least 19, and since they form a one-dimensional family the generic Picard group has rank 19.

In the second half of the paper we discuss how one can find a basis for a subgroup of finite index of the generic Picard group for the five families from [8] and for five further monomial deformations of Delsarte quartic surfaces. For all ten families we determine $H^{2}\left(Y_{\psi}\right)^{G}, H^{2}\left(Y_{\psi}\right)^{G_{\max }}$ and $C$ as vector spaces with Frobenius action Moreover, we find curves generating $C$ in each of the ten cases. For two families we have that $W_{\psi}$ is zero-dimensional. For six of the remaining eight families we manage to find curves, whose classes in cohomology generate $W_{\psi}$.

In the next section we prove our generalisation of the result from [8]. In the third section we discuss the quartic surface case. In Appendix A we give explicit equations for bitangents to certain particular quartic plane curves. These equations can be used to find explicit curves, generating $W_{\psi}$.

\section{Delsarte hypersurface}

Fix an integer $n \geq 2$ and fix a finite field $\mathbf{F}_{q}$.

Definition 2.1. An invertible matrix $A:=\left(a_{i, j}\right)_{0 \leq i, j \leq n}$, such that all entries are nonnegative integers is called a coefficient matrix if all entries of $A^{-1}(1, \ldots, 1)^{\mathrm{T}}$ are positive and each column of $A$ contains a zero.

In that case let $d$ be an integer such that $B:=d A^{-1}$ has integral coefficients. We call $B$ the map matrix. We call $B(1, \ldots, 1)^{\mathrm{T}}$ the weight vector, which we denote by $\mathbf{w}:=\left(w_{0}, \ldots, w_{n}\right)$.

A vector $\mathbf{a}:=\left(a_{0}, \ldots, a_{n}\right)$ consisting of nonnegative integers such that $\sum_{i=0}^{n} w_{i} a_{i}=d$ holds and such that all entries of $\mathbf{a} A^{-1}$ are nonnegative is called a deformation vector.

Definition 2.2. Fix a pair $(A, \mathbf{a})$ consisting of coefficient matrix and a deformation vector a. Assume that $\operatorname{gcd}(q, d)=1$. Then we call $(A, \mathbf{a})$ Delsarte deformation data of length $n$.

Let $(A, \mathbf{a})$ be Delsarte deformation data of length $n$. Let

$$
X_{\lambda}:=Z\left(\sum_{i=0}^{n} \prod_{j=0}^{n} x_{j}^{a_{i, j}}+\lambda \prod_{i=0}^{n} x_{i}^{a_{i}}\right)
$$


be the corresponding one-parameter family of hypersurfaces of weighted degree $d$ in the weighted projective space $\mathbf{P}\left(w_{0}, \ldots, w_{n}\right)$.

Denote with $\left(b_{0}, \ldots, b_{n}\right)$ the entries of $\mathbf{a} B$. Let $Y_{\lambda}$ be

$$
Z\left(\sum_{i=0}^{n} y_{i}^{d}+\lambda \prod_{i=0}^{n} y_{i}^{b_{i}}\right) \subset \mathbf{P}^{n} .
$$

Remark 2.3. Our definition of $\mathbf{w}$ may lead to choices of the $w_{i}$ such that the gcd of $\left(w_{0}, \ldots, w_{n}\right)$ is larger than one. The choice of the $w_{i}$ is such that the weighted degree of the polynomial defining $X_{\lambda}$ equals the degree of $Y_{\lambda}$.

We have a $(\mathbf{Z} / d \mathbf{Z})^{n}$-action on $\mathbf{P}^{n}$ induced by

$$
\left(g_{1}, \ldots, g_{n}\right)\left(x_{0}: x_{1}: \cdots: x_{n}\right):=\left(x_{0}: \zeta^{g_{1}} x_{1}: \zeta^{g_{2}} x_{2}: \cdots: \zeta^{g_{n}} x_{n}\right) \text {, }
$$

with $\zeta$ a fixed primitive $d$-th root of unity. The subgroup $G$ defined by $\sum_{i=1}^{n} g_{i} b_{i} \equiv 0 \bmod d$ acts on $Y_{\lambda}$.

The rational map $\mathbf{P}^{n} \rightarrow \mathbf{P}(\mathbf{w})$ given by

$$
\left(y_{0}, y_{1}, \ldots, y_{n}\right) \mapsto\left(\prod_{i=0}^{n} y_{i}^{b_{0, i}}, \prod_{i=0}^{n} y_{i}^{b_{1, i}}, \ldots, \prod_{i=0}^{n} y_{i}^{b_{n, i}}\right)
$$

induces a rational map $Y_{\lambda} \rightarrow X_{\lambda}$. This rational map is Galois (i.e., the corresponding extension of function fields is Galois) and the Galois group is a subgroup of $G$.

In particular, if all the $b_{i, j}$ are nonnegative then this rational map is a morphism. (This map was used by Shioda [20] to give an algorithm to calculate the Picard number of a Delsarte surface in $\mathbf{P}^{3}$.)

Lemma 2.4. The hypersurface $X_{0}$ is irreducible.

Proof. Each column of $A$ contains a zero by the definition of coefficient matrix. Hence $x_{k}$ does not divide

$$
\sum_{i=0}^{n} \prod_{j=0}^{n} x_{j}^{a_{i, j}}
$$

for any $k$. Hence for every irreducible component of $X_{0}$ the points such that all coordinates are nonzero are dense, and these latter points are in the image of $Y_{0}$. This implies that every irreducible component of $X_{0}$ is the closure of an irreducible component of the image of $Y_{0}$. Since $n>1$ it follows that $Y_{0}$ is irreducible and hence $X_{0}$ is irreducible.

Definition 2.5. We call $X_{0}$ the Delsarte hypersurface associated with $A$ and $X_{\lambda}$ the one-dimensional monomial deformation associated with $(A, \mathbf{a})$. If, moreover, $X_{0}$ is quasismooth then we call $X_{0}$ invertible hypersurface.

Example 2.6. Consider

$$
x_{0}^{4}+x_{1}^{4}+x_{2}^{3} x_{3}+x_{3}^{3} x_{2}+\lambda x_{0} x_{1} x_{2} x_{3} .
$$

Then

$$
A=\left(\begin{array}{llll}
4 & 0 & 0 & 0 \\
0 & 4 & 0 & 0 \\
0 & 0 & 3 & 1 \\
0 & 0 & 1 & 3
\end{array}\right) \quad \text { and } \quad \mathbf{a}=(1,1,1,1)
$$


We have that

$$
B=\left(\begin{array}{cccc}
2 & 0 & 0 & 0 \\
0 & 2 & 0 & 0 \\
0 & 0 & 3 & -1 \\
0 & 0 & -1 & 3
\end{array}\right) \quad \text { and } \quad \mathbf{w}=(2,2,2,2)
$$

In particular, we have that this family is birational a quotient of

$$
x_{0}^{8}+x_{1}^{8}+x_{2}^{8}+x_{3}^{8}+\lambda\left(x_{0} x_{1} x_{2} x_{3}\right)^{2} .
$$

The group $G$ is generated by the automorphisms

$$
\left(x_{0}, x_{1}, x_{2}, x_{3}\right) \mapsto\left(x_{0},-x_{1}, x_{2}, x_{3}\right) \quad \text { and } \quad\left(x_{0}, x_{1}, x_{2}, x_{3}\right) \mapsto\left(x_{0}, x_{1}, \zeta^{3} x_{2}, \zeta x_{3}\right),
$$

with $\zeta$ a primitive 8 -th root of unity.

Definition 2.7. A hypersurface $X=V(f) \subset \mathbf{P}^{n}$ is in general position if

$$
V\left(x_{0} \frac{\partial}{\partial x_{0}} f, \ldots, x_{n} \frac{\partial}{\partial x_{n}} f\right)
$$

is empty. Equivalently, $X$ is smooth and for any subset $\left\{i_{1}, \ldots, i_{c}\right\} \subset\{0,1, \ldots, n\}$ we have that

$$
X \cap V\left(x_{i_{1}}\right) \cap \cdots \cap V\left(x_{i_{c}}\right)
$$

is also smooth.

Lemma 2.8. If $Y_{\lambda}$ is smooth then $Y_{\lambda}$ is in general position.

Proof. Suppose we intersect $Y_{\lambda}$ with $x_{i_{1}}=\cdots=x_{i_{c}}=0$. If some $b_{i_{j}}$ is nonzero then the intersection is a Fermat hypersurface in $\mathbf{P}^{n-c}$ and is smooth. If all $b_{i_{j}}$ are zero then we can do the following: After a change of coordinates we may assume that $\left\{i_{1}, \ldots, i_{c}\right\}=\{0,1, \ldots, c-1\}$. We now have that $Y_{\lambda}$ is the zero set of

$$
\sum_{i=0}^{c-1} x_{i}^{d}+h\left(x_{c}, \ldots, x_{n}\right)
$$

for some $h \in \mathbf{F}_{q}\left[x_{c}, \ldots, x_{n}\right]$. From $\operatorname{gcd}(q, d)=1$ it follows that the singular points of the intersection $V\left(x_{0}, \ldots, x_{c-1}, h\right)$ are in one-to-one correspondence with the singular points of $Y_{\lambda}$. Hence $V\left(x_{0}, \ldots, x_{c-1}, h\right)$ is smooth.

Recall that we started with a hypersurface $X_{\lambda} \subset \mathbf{P}(\mathbf{w})$ and constructed a hypersurface $Y_{\lambda} \subset \mathbf{P}^{n}$, such that $X_{\lambda}$ is birational to a quotient of $Y_{\lambda}$. Denote with $U_{\lambda}:=\mathbf{P}(\mathbf{w}) \backslash X_{\lambda}$ and $V_{\lambda}:=\mathbf{P}^{n} \backslash Y_{\lambda}$ be the respective complements.

Denote now with $(\mathbf{P}(\mathbf{w}))^{*}, U_{\lambda}^{*}, V_{\lambda}^{*}, X_{\lambda}^{*}, Y_{\lambda}^{*}$, etc. the original variety minus the intersection with $Z\left(x_{0} \ldots x_{n}\right)$ or $Z\left(y_{0} \ldots y_{n}\right)$, the union of the coordinate hyperplanes. We have that the quotient map $\mathbf{P}^{n} \rightarrow \mathbf{P}(\mathbf{w})$ defines surjective morphisms $\left(\mathbf{P}^{n}\right)^{*} \rightarrow \mathbf{P}(\mathbf{w})^{*}, Y_{\lambda}^{*} \rightarrow X_{\lambda}^{*}, V_{\lambda}^{*} \rightarrow U_{\lambda}^{*}$.

There is a second quotient map $\mathbf{P}^{n} \rightarrow \mathbf{P}(\mathbf{w})$ given by $\left(z_{0}: \cdots: z_{n}\right) \rightarrow\left(z_{0}^{w_{0}}: \cdots: z_{n}^{w_{n}}\right)$. This map is a morphism and is a ramified Galois covering. Denote with $H$ the corresponding Galois group. Let $\tilde{X}_{\lambda}$ be the pull back of $X_{\lambda}$ and let $\tilde{U}_{\lambda}$ be the pull back of $U_{\lambda}$.

Fix now a lift $\mu \in \mathbf{Q}_{q}$ of $\lambda$. Then we can define $F_{\mu}, \tilde{U}_{\mu}, V_{\mu}, \tilde{X}_{\mu}, Y_{\mu}$ similarly as above. If $y_{0}, \ldots, y_{n}$ are projective coordinates on $\mathbf{P}^{n}$ then let $\Omega$ be

$$
\left(\prod_{i=0}^{n} y_{i}\right)\left(\sum_{i=0}^{n}(-1)^{i} \frac{d y_{0}}{y_{0}} \wedge \cdots \wedge \frac{\widehat{d y_{i}}}{y_{i}} \wedge \cdots \wedge \frac{d y_{n}}{y_{n}}\right) \text {. }
$$

We recall now some standard notation used to study the cohomology of a hypersurface complement in $\mathbf{P}^{n}$. 
Notation 2.9. Let $\mathbf{m}=\left(m_{0}, \ldots, m_{n}\right)$ be $(n+1)$-tuple of positive integers, such that $\sum_{i=0}^{n} m_{i}=t d$ for some positive integer $t$. Then

$$
\tilde{\omega}_{\mathbf{m}}:=\frac{\prod_{i=0}^{n} x_{i}^{m_{i}-1}}{\left(F_{A, \mu}\left(x_{0}^{w_{0}}, \ldots, x_{n}^{w_{n}}\right)\right)^{t}} \Omega
$$

is an $n$-form on the complement $\tilde{U}_{\mu}$ of $\tilde{X}_{\mu}$. If we allow the $m_{i}$ and $t$ to be arbitrary integers such that the equality $\sum_{i=0}^{n} m_{i}=t d$ holds then $\tilde{\omega}_{\mathbf{m}}$ is a form on $\tilde{U}_{\mu}^{*}$.

Let $\mathbf{m}=\left(m_{0}, \ldots, m_{n}\right)$ be $(n+1)$-tuple of positive integers, such that $\sum_{i=0}^{n} m_{i}=t d$ holds for some positive integer $t$. Let $D$ be the diagonal matrix $d I_{n+1}$. Then

$$
\omega_{\mathbf{m}}:=\frac{\prod_{i=0}^{n} y_{i}^{m_{i}-1}}{F_{D, \mu}^{t}} \Omega
$$

is an $n$-form on the complement $V_{\mu}$ of $Y_{\mu}$. If we allow the $m_{i}$ and $t$ to be arbitrary integers such that the equality $\sum_{i=0}^{n} m_{i}=t d$ then $\omega_{\mathbf{m}}$ is a form on $V_{\mu}^{*}$.

The following result seems to be known to the experts, but we include it for the reader's convenience:

Lemma 2.10. There exists a finite set $S \subset \mathbf{Q}_{q}$ such that $0 \notin S$ and for all $\mu \in \mathbf{Q}_{q} \backslash S$ we have that

$$
\mathcal{B}:=\left\{\omega_{\mathbf{m}}: 0<m_{i}<d \text { for } i=0, \ldots, n \text { and } \sum_{i=0}^{n} m_{i} \equiv 0 \bmod d\right\}
$$

is a basis for $H_{\mathrm{dR}}^{n}\left(V_{\mu}, \mathbf{Q}_{q}\right)$.

Similarly, there exists a finite set $S^{*}$ such that $0 \notin S^{*}$ and for all $\mu \in \mathbf{Q}_{q} \backslash S^{*}$ we have that

$$
\mathcal{B}^{*}:=\left\{\omega_{\mathbf{m}}: 0 \leq m_{i}<d \text { for } i=0, \ldots, n \text { and } \sum_{i=0}^{n} m_{i} \equiv 0 \bmod d\right\}
$$

is a basis for $H_{\mathrm{dR}}^{n}\left(V_{\mu}^{*}, \mathbf{Q}_{q}\right)$.

Proof. The forms $\omega_{\mathbf{m}}$, such that $m_{i} \geq 1$ for $i=0, \ldots, n$ generate the de Rham cohomology group $H_{\mathrm{dR}}^{n}\left(V_{\mu}\right)$. By differentiating certain particular $(n-1)$-forms on $V_{\mu}$ we have that the following relation in $H_{\mathrm{dR}}^{n}\left(V_{\mu}\right)$

$$
\frac{G_{y_{i}}}{F^{t}} \Omega=\frac{t G F_{y_{i}}}{F^{t+1}} \Omega
$$

for any form $G \in \mathbf{Q}_{q}\left[y_{0}, \ldots, y_{n}\right]_{t d-n}$. (This is the so-called Griffiths-Dwork method to reduce forms in cohomology.)

For $\mu=0$ we have that $F_{y_{i}}=d y_{i}^{d-1}$. Using (2.1) we find the relation

$$
\frac{y_{0}^{m_{0}} G\left(y_{1}, \ldots, y_{n}\right)}{F_{0}^{t+1}} \Omega=\frac{\left(m_{0}-d+1\right) y_{0}^{m_{0}-d} G\left(y_{1}, \ldots, y_{n}\right)}{t F_{0}^{t}} \Omega
$$


and similar relations for the other $y_{i}$. In this way we can reduce forms such that all exponents are at least 0 and at most $d-1$. However, if an exponent equals $d-1$ then this relation yields that the class is zero in cohomology. In particular, the $\omega_{\mathbf{m}}$ with $0<m_{i}<d$ for $i=0, \ldots, n$ and $\sum_{i=0}^{n} m_{i} \equiv 0 \bmod d$ generate $H_{\mathrm{dR}}^{n}\left(V_{0}\right)$. Griffiths [11] showed that the relations of type (2.1) generate all relations and hence $\mathcal{B}$ is a basis for $H_{\mathrm{dR}}^{n}\left(V_{0}\right)$. If $X_{\mu}$ is smooth then the dimension of $H_{\mathrm{dR}}^{n}\left(V_{\mu}\right)$ is independent of $\mu$ and it is then straightforward to check that there are at most finitely many choices of $\mu$ for which $X_{\mu}$ is smooth and $\mathcal{B}$ is not a basis for $H_{\mathrm{dR}}^{n}\left(V_{\mu}\right)$.

We now prove the statement on $H_{\mathrm{dR}}^{n}\left(V_{\mu}^{*}\right)$. Note that if $X_{\mu}$ is smooth then by Lemma 2.8 it is in general position. Therefore the dimension of $H_{\mathrm{dR}}^{n}\left(V_{\mu}^{*}\right)$ is independent of $\mu$. Hence it suffices to show that $\mathcal{B}^{*}$ is a basis for $H_{\mathrm{dR}}^{n}\left(V_{0}^{*}\right)$. Again we have relations of type (2.1), but now we may take $G \in \mathbf{Q}_{q}\left[y_{0}, y_{0}^{-1}, \ldots, y_{n}, y_{n}^{-1}\right]_{t d-n}$. If the exponent of a variable is at most -2 then we can use the relations of the shape (2.2) to increase the exponent of this variable. However, if the exponent equals -1 we cannot do this, because then we would have to divide by zero in (2.2). In this way we obtain that $\mathcal{B}^{*}$ generates $H_{\mathrm{dR}}^{n}\left(V_{0}^{*}\right)$. Moreover, as in the above case there are no further relations and $\mathcal{B}^{*}$ is a basis.

Remark 2.11. The $\mu$ for which $\mathcal{B}$ is not a basis can be determined by the methods of $[18$, Section 3].

Remark 2.12. Denote with $H_{\mathrm{MW}}^{n}\left(V_{\lambda}\right)$ the $n$-th Monsky-Washnitzer cohomology of $V_{\lambda}$. The Monsky-Washnitzer cohomology is essentially the cohomology of the tensor product of de Rham complex of a lift of $V_{\lambda}$ to characteristic zero with a weakly complete finitely generated algebra $A^{\dagger}$. The Frobenius action on the cohomology is induced by a lift of Frobenius to $A^{\dagger}$. For more details see [21, Theorem 2.4.5]. In that paper it is shown that two different lifts of $V_{\lambda}$ yield isomorphic complexes and two choices of lifts of Frobenius yield homotopic maps on the complexes. In particular, $H_{\mathrm{MW}}^{n}\left(V_{\lambda}\right)$ is independent of the choices made.

Let $\mu \in \mathbf{Q}_{q}$ be a lift of $\lambda$. One choice of a lift of $V_{\lambda}$ to characteristic zero is $V_{\mu}$, and the construction of the Monsky-Washnitzer cohomology yields a natural map $H_{\mathrm{dR}}^{n}\left(V_{\mu}\right) \rightarrow H_{\mathrm{MW}}^{n}\left(V_{\lambda}\right)$ of $\mathbf{Q}_{q}$-vector spaces. If $Y_{\lambda}$ is smooth then the is an isomorphism by [1]. Since $V_{\lambda} \subset \mathbf{P}^{n}$ is affine and smooth we have an isomorphism $H_{\mathrm{MW}}^{n}\left(V_{\lambda}\right) \cong H_{\text {rig }}^{n}\left(V_{\lambda}\right)$ by [2], where the latter group is rigid cohomology. Since there are infinitely many lifts $\mu$ of $\lambda$ we can always choose a lift $\mu$ such that $\mathcal{B}$ is a basis for $H_{\mathrm{dR}}^{n}\left(V_{\mu}\right)$ and thereby yielding a basis for $H_{\mathrm{MW}}^{n}\left(V_{\lambda}\right)$.

If $Y_{\lambda}$ is smooth then using Lemma 2.8 we find that $V_{\lambda}^{*}$ is the complement of a normal crossing divisor. In particular, we can apply [1] and find a natural isomorphism $H_{\mathrm{MW}}^{n}\left(V_{\lambda}^{*}\right) \cong H_{\mathrm{dR}}^{n}\left(V_{\mu}^{*}\right)$. As above, we have an isomorphism $H_{\mathrm{MW}}^{n}\left(V_{\lambda}^{*}\right) \cong H_{\text {rig }}^{n}\left(V_{\lambda}^{*}\right)$ and we identified a basis for $H_{\text {rig }}^{n}\left(V_{\lambda}^{*}\right)$.

Remark 2.13. The action of $G$ lifts to characteristic zero. The forms $\omega_{\mathbf{m}}$ are eigenvectors for $g^{*}$ each element $g \in G$. Hence the $G$-invariant ones span $H_{\mathrm{MW}}^{n}\left(V_{\lambda}\right)^{G}$.

If $Y_{\lambda}$ is singular then by the definition of Monsky-Washnitzer cohomology we have that $H_{\mathrm{MW}}^{n}\left(V_{\lambda}\right)$ is generated by expressions

$$
\sum_{\mathbf{m}=\left(m_{0}, \ldots, m_{n}\right), m_{i} \geq 1} a_{\mathbf{m}} \omega_{\mathbf{m}}
$$

such that there exists $c_{1}, c_{2} \in \mathbf{Q}$ with $c_{1}>0$ and $v\left(a_{\mathbf{m}}\right) \geq c_{1}\left(\sum_{i=0}^{n} m_{i}\right)+c_{2}$. The space $H_{\mathrm{MW}}^{n}\left(V_{\lambda}^{*}\right)$ is generated by expression

$$
\sum_{\mathbf{m}=\left(m_{0}, \ldots, m_{n}\right), m_{i} \geq-N} a_{\mathbf{m}} \omega_{\mathbf{m}}
$$


such that there exists $c_{1}, c_{2} \in \mathbf{Q}$ with $c_{1}>0$ and $v\left(a_{\mathbf{m}}\right) \geq c_{1}\left(\sum_{i=0}^{n} m_{i}\right)+c_{2}$. The $G$-invariant subspaces are generated by similar sums, but in which only the $G$-invariant $\omega_{\mathbf{m}}$ occur.

Remark 2.14. If one wants to study the Frobenius matrix by using the differential equations, like in [12] or in [8] then one needs to be more careful in lifting $V_{\lambda}$ to characteristic zero. In [12] one has to take $\mu$ to be the Teichmüller lift of $\lambda$. The reason for this, is that a priori Frobenius maps $H^{i}\left(U_{\mu}^{q}\right)$ to $H^{i}\left(U_{\mu}\right)$. To have an operator on $H^{n}\left(U_{\mu}\right)$ we need that $\mu^{q}=\mu$. If one works directly with Frobenius on Monsky-Washnitzer chomology then this constraint on $\mu$ does not exist.

From now on we use $H^{i}$ and $H_{c}^{i}$ to indicate rigid cohomology respectively rigid cohomology with compact support.

By [16, Proposition 2.1] we have canonical isomorphisms

$$
H_{c}^{i}\left(U_{\lambda}^{*}\right) \cong H_{c}^{i}\left(V_{\lambda}^{*}\right)^{G} \quad \text { and } \quad H_{c}^{i}\left(U_{\lambda}^{*}\right) \cong H_{c}^{i}\left(\tilde{U}_{\lambda}^{*}\right)^{H} .
$$

We want to compare the cohomology of $H_{c}^{n}\left(V_{\lambda}\right)^{G}$ with the cohomology of $H_{c}^{n}\left(U_{\lambda}\right)$. However, $U_{\lambda}$ may be singular, hence we work with $H_{c}^{n}\left(\tilde{U}_{\lambda}\right)^{H}$ instead. Using Poincaré duality it suffices to compare $H^{n}\left(V_{\lambda}\right)^{G}$ with $H^{n}\left(\tilde{U}_{\lambda}\right)^{H}$ instead. Since both varieties are smooth and affine we can identify their rigid cohomology groups with their Monsky-Washnitzer cohomology groups. We will do this in order to prove:

Proposition 2.15. Suppose that $H^{n}\left(V_{\lambda}\right) \rightarrow H^{n}\left(V_{\lambda}^{*}\right)$ is injective. Then $H^{n}\left(V_{\lambda}\right)^{G}$ is a quotient of $H^{n}\left(\tilde{U}_{\lambda}\right)^{H}$. In particular, the characteristic polynomial of Frobenius acting on $H_{c}^{n}\left(V_{\lambda}\right)^{G}$ is in $\mathbf{Q}[T]$ and divides the characteristic polynomial of Frobenius acting on $H_{c}^{n}\left(U_{\lambda}\right)$.

Proof. Since $H^{n}\left(V_{\lambda}\right) \rightarrow H^{n}\left(V_{\lambda}^{*}\right)$ is injective we have by [3] that the Poincaré dual of this map is surjective, and therefore that $H_{c}^{n}\left(V_{\lambda}\right)^{G}$ is a quotient of $H_{c}^{n}\left(V_{\lambda}^{*}\right)^{G}$. This implies that $H_{c}^{n}\left(V_{\lambda}\right)^{G}$ is also a quotient of $H_{c}^{n}\left(\tilde{U}_{\lambda}^{*}\right)^{H}$. Hence it suffices to show that the kernel of natural map $H_{c}^{n}\left(\tilde{U}_{\lambda}^{*}\right) \rightarrow H_{c}^{n}\left(\tilde{U}_{\lambda}\right)$ is mapped to zero in $H_{c}^{n}\left(V_{\lambda}\right)^{G}$. Using Poincaré duality we can consider $H^{n}\left(V_{\lambda}\right)^{G}$ as a subspace of $H^{n}\left(\tilde{U}_{\lambda}^{*}\right)^{H}$. It suffices to show that $H^{n}\left(V_{\lambda}\right)^{G}$ is in the image of $H^{n}\left(\tilde{U}_{\lambda}\right)$.

A form $\omega_{\mathbf{k}}$ is in $H^{n}\left(V_{\lambda}\right)^{G}$ if and only if there is a monomial type $\mathbf{m}_{0}$ such that $\mathbf{k}=\mathbf{m}_{0} B$. We identified $H^{n}\left(V_{\lambda}\right)^{G}$ with a subspace of $H^{n}\left(\tilde{U}_{\lambda}^{*}\right)^{H}$. The class of $\omega_{\mathbf{k}}$ is identified with $\tilde{\omega}_{\mathbf{m}}$ where $\mathbf{m}=\mathbf{m}_{0}\left(\operatorname{diag}\left(w_{0}, \ldots, w_{n}\right)\right)$.

The entries of $\mathbf{m}$ are integers, which may be nonpositive. If all entries of $\mathbf{m}$ are positive then $\omega_{\mathbf{m}}$ is in the image of $H^{n}\left(\tilde{U}_{\lambda}\right)^{H}$. Recall that $B=d A^{-1}$ and therefore $\mathbf{m}_{0}=\mathbf{k} \frac{1}{d} A$. Since $\mathbf{k}$ has positive entries, $A$ has positive entries and no zero column it follows that also the entries of $\mathbf{m}_{0}$ are positive and therefore all entries of $\mathbf{m}$ are also positive. This yields the first statement.

To prove the second statement. By [16, Lemma 4.3] it follows that $H_{c}^{n}\left(V_{\lambda}\right)^{G}$ is Frobenius invariant and the characteristic polynomial is in $\mathbf{Q}[T]$. Using Poincaré duality we find that $H_{c}^{n}\left(V_{\lambda}\right)^{G}$ is a subspace of $H_{c}^{n}\left(\tilde{U}_{\lambda}\right)^{H}$. As explained above, the latter space is isomorphic with $H_{c}^{n}\left(U_{\lambda}\right)$.

Definition 2.16. Fix Delsarte deformation data $\left(A_{1}, \mathbf{a}_{1}\right), \ldots,\left(A_{t}, \mathbf{a}_{t}\right)$ of length $n$. We say that they have a common cover if for every $i, j$ we have that $\mathbf{a}_{i}^{\mathrm{T}} A_{i}^{-1}$ and $\mathbf{a}_{j}^{\mathrm{T}} A_{j}^{-1}$ are proportional.

Example 2.17. Take the following five matrices

$$
\left(\begin{array}{llll}
4 & 0 & 0 & 0 \\
0 & 4 & 0 & 0 \\
0 & 0 & 4 & 0 \\
0 & 0 & 0 & 4
\end{array}\right), \quad\left(\begin{array}{llll}
4 & 0 & 0 & 0 \\
0 & 4 & 0 & 0 \\
0 & 0 & 3 & 1 \\
0 & 0 & 1 & 3
\end{array}\right), \quad\left(\begin{array}{llll}
3 & 1 & 0 & 0 \\
1 & 3 & 0 & 0 \\
0 & 0 & 3 & 1 \\
0 & 0 & 1 & 3
\end{array}\right), \quad\left(\begin{array}{llll}
4 & 0 & 0 & 0 \\
0 & 3 & 1 & 0 \\
0 & 0 & 3 & 1 \\
0 & 1 & 0 & 3
\end{array}\right), \quad\left(\begin{array}{llll}
3 & 1 & 0 & 0 \\
0 & 3 & 1 & 0 \\
0 & 0 & 3 & 1 \\
1 & 0 & 0 & 3
\end{array}\right) .
$$


In each case we take $(1,1,1,1)^{\mathrm{T}}$ as the deformation vector then the $\left(A_{i}, \mathbf{a}_{i}\right)$ have a common cover.

Suppose now that $\left(A_{1}, \mathbf{a}_{1}\right), \ldots,\left(A_{t}, \mathbf{a}_{t}\right)$ have a common cover. Let $d$ be the smallest positive integer such that $d A_{i}^{-1}$ has integral coefficients for all $i$. The sum of the entries of $\mathbf{b}_{i}=\mathbf{a}_{i}\left(d A_{i}^{-1}\right)$ equals $d$. By assumption we have that for each $i$ and $j$ the vectors $\mathbf{b}_{i}$ and $\mathbf{b}_{j}$ are proportional, hence these vectors coincide and we denote this common vector with $\mathbf{b}$.

Denote with $b_{j}$ the entries of $\mathbf{b}$. Denote with $X_{i, \lambda}$ the family associated with $\left(A_{i}, \mathbf{a}_{i}\right)$. Then $X_{i, \lambda}$ is birational to a quotient of

$$
Y_{\lambda}: \sum_{i=0}^{n} y_{i}^{d}+\lambda \prod_{i=0}^{n} y_{i}^{b_{i}}
$$

At the beginning of this section we gave an explicit description of this map. From that description it follows that $Y_{\lambda} \rightarrow X_{i, \lambda}$ is defined whenever all the $y_{i}$ are nonzero.

We can now apply Proposition 2.15 to the above setup and we find directly that:

Theorem 2.18. Let $\left(A_{1}, \mathbf{a}_{1}\right), \ldots,\left(A_{t}, \mathbf{a}_{t}\right)$ be Delsarte deformation data of length $n$ with a common cover. Denote with $X_{i, \lambda}$ be the corresponding families of Delsarte hypersurfaces and with $Y_{\lambda}$ the common cover. Let $G_{i}$ be the Galois group of the function field extension corresponding to the rational map $Y_{\lambda} \rightarrow X_{i, \lambda}$. Then the automorphisms in $G_{i}$ extend to automorphisms of $Y_{\lambda}$. Identify $G_{i}$ with the corresponding subgroup of $\operatorname{Aut}\left(Y_{\lambda}\right)$. Let $G=G_{1} \cdot G_{2} \ldots \ldots G_{t} \subset \operatorname{Aut}\left(Y_{\lambda}\right)$.

Suppose that $H^{n}\left(V_{\lambda}\right) \rightarrow H^{n}\left(V_{\lambda}^{*}\right)$ is injective. Then for each $i=1, \ldots, t$ we have that $H_{c}^{n}\left(V_{\lambda}\right)^{G}$ is a quotient of $H_{c}^{n}\left(U_{i, \lambda}\right)$. In particular, the characteristic polynomial of Frobenius on $H^{n-1}\left(Y_{\lambda}\right)^{G}$ is in $\mathbf{Q}[T]$ and is a common factor of the characteristic polynomials of Frobenius acting on $H^{n-1}\left(X_{i, \lambda}\right)$.

Remark 2.19. Recall that in order to be Delsarte deformation data we need that $\operatorname{gcd}(q,(n+$ 1) $\left.\operatorname{det}\left(A_{i}\right)\right)=1$ for all $i$.

Remark 2.20. If $Y_{\lambda}$ is smooth then it is in general position by Lemma 2.8.

The map $H^{n-1}\left(Y_{\lambda}\right) \rightarrow H^{n-1}\left(Y_{\lambda}^{*}\right)$ is injective if $n-1$ is even, and has a kernel if $n-1$ is odd, and this kernel is generated by the hyperplane class, see [12, Theorem 1.19]. The residue map identifies $H^{n}\left(V_{\lambda}\right)$ with the primitive part of the cohomology of $H^{n-1}\left(Y_{\lambda}\right)$. In particular, the composition $H^{n}\left(V_{\lambda}\right) \rightarrow H^{n-1}\left(Y_{\lambda}^{*}\right)$ is injective independent of the parity of $n$. From the diagram on [12, p. 79] it follows that the latter map factors through $H^{n}\left(V_{\lambda}^{*}\right)$. In particular, $H^{n}\left(V_{\lambda}\right) \rightarrow H^{n}\left(V_{\lambda}^{*}\right)$ is injective. Hence we can apply the above proposition if $Y_{\lambda}$ is smooth. The values of $\lambda$ for which $Y_{\lambda}$ is singular can be determined from the formula [15, Lemma 3.7].

To conclude that there is a common factor of the zeta function is more complicated in general. The zeta function is a quotient of products of characteristic polynomials of Frobenius and there may be some cancellation in this quotient. However, if we make the extra assumptions that each $X_{i, \lambda}$ is a hypersurface in $\mathbf{P}^{n}$ (i.e., for each $i$ we have that $\mathbf{w}=(k, \ldots, k)$ for some $k \in \mathbf{Z}_{>0}$ ) and we consider only values of $\lambda$ for which $X_{\lambda}$ is smooth then we have that

$$
\left(Z\left(X_{i, \lambda}, T\right) \prod_{j=0}^{n-1}\left(1-q^{j} T\right)\right)^{(-1)^{n}}=\operatorname{det}\left(I-T \text { Frob }^{*}: H_{c}^{n}\left(U_{i, \lambda}\right)\right) .
$$

From the smoothness of $X_{i, \lambda}$ it follows that the eigenvalues of Frobenius on $H_{c}^{n}\left(U_{i, \lambda}\right)$ have absolute value $q^{n-1 / 2}$, hence there is no cancellation in this formula and we obtain: 
Corollary 2.21. Let $\left(A_{1}, \mathbf{a}_{1}\right), \ldots,\left(A_{t}, \mathbf{a}_{t}\right)$ be Delsarte deformation data of length $n$ with a common cover. Denote with $X_{i, \lambda}$ be the corresponding families of Delsarte hypersurfaces and with $Y_{\lambda}$ the common cover. Let $G_{i}$ be the Galois group of the function field extension corresponding to $Y_{\lambda} \rightarrow X_{i, \lambda}$. Let $G=G_{1} \cdot G_{2} \ldots G_{t} \subset \operatorname{Aut}\left(Y_{\lambda}\right)$.

Suppose that for each $i$ we have $\mathbf{P}(\mathbf{w})=\mathbf{P}^{n}$. Moreover, suppose that $Y_{\lambda}$ and each $X_{i, \lambda}$ is smooth. Then the characteristic polynomial of Frobenius on $H^{n-1}\left(Y_{\lambda}\right)_{\text {prim }}^{G}$ is in $\mathbf{Q}[T]$ and divides the polynomial

$$
\left(Z\left(X_{i, \lambda}, T\right) \prod_{j=0}^{n-1}\left(1-q^{j} T\right)\right)^{(-1)^{n}}
$$

Remark 2.22. A complex hypersurface with quotient singularities is a Q-homology manifold and satisfies Poincaré duality. The existence of Poincaré duality is sufficient to obtain both the vanishing statement $H_{c}^{i}\left(\mathbf{P}(\mathbf{w}) \backslash X_{i, \lambda}\right)=0$ for $i \neq n, 2 n$ as well as for the purity statement on $H_{c}^{n}\left(\mathbf{P}(\mathbf{w}) \backslash X_{i, \lambda}\right)$.

Hence if Poincaré duality would hold for the rigid cohomology of varieties with (tame) quotient singularities over finite fields then we could extend the above corollary to the case where $X_{i, \lambda}$ is a quasi-smooth hypersurface.

We would like to compare our factor with the factor found in [8]. The groups $G$ and $G^{\prime}$ consists of torus automorphisms of $Y_{\lambda}$. Let $G_{\max }$ be the group of torus automorphism of $Y_{\lambda}$. Then $G_{\max }$ is an abelian group. A torus automorphism $g \in G_{\max }$ sends $Y_{\lambda}^{*}$ to itself, and descents to an automorphism of $X_{\lambda}^{*} \cong Y_{\lambda}^{*} / G$. Hence the quotient group $G_{\max } / G$ acts on $X_{\lambda}^{*}$.

Since the quotient map is given by $n+1$ monomials we have that a torus automorphism descends to a torus automorphism of $X_{\lambda}^{*}$ and $U_{\lambda}^{*}$. Any torus automorphism can be extended to $\mathbf{P}(\mathbf{w})$, leaving $X_{\lambda}$ invariant. Hence we have an action of $G_{\max } / G$ on $H^{n}\left(U_{\lambda}\right)$ and on $H_{c}^{n}\left(U_{\lambda}\right)$. It is straightforward to check that $G_{\max } / G \cong \operatorname{SL}\left(F_{A}\right)$, where $\operatorname{SL}\left(F_{A}\right)$ is the group introduced in [8], and that both groups act the same.

The factor from [8] is constructed as follows: The authors identify a subspace of the Dwork cohomology group $H_{\text {Dwork }}^{n}\left(U_{\lambda}\right)^{\mathrm{SL}\left(F_{A}\right)}$, whose dimension equals the order of the Picard-Fuchs equation of $X_{\lambda}$ and which is invariant under Frobenius. They show that the characteristic polynomial $R_{\lambda}^{\prime}$ of Frobenius on this subspace is in $K[T]$ for some number field $K$, which can be taken Galois over $\mathbf{Q}$ and then take $R_{\lambda}$ the be the least common multiple of the Galois conjugates of $R_{\lambda}^{\prime}$.

To compare this polynomial with the factor constructed above, we will start by reconsidering $R_{\lambda}^{\prime}$, i.e., we will show that it is just the characteristic polynomial of Frobenius acting on

$$
H_{c}^{n}\left(V_{\lambda}\right)^{G_{\max }} .
$$

Then [16, Lemma 4.3] implies that $R_{\lambda}^{\prime} \in \mathbf{Q}[T]$ and that $R_{\lambda}=R_{\lambda}^{\prime}$.

We start by calculating the dimension of $H_{c}^{n}\left(V_{\lambda}\right)^{G_{\max }}$.

Lemma 2.23. Suppose that $X_{\lambda}$ is Calabi-Yau, i.e., $\sum w_{i}=d$ and suppose that $Y_{\lambda}$ is smooth. Then the dimension of $H_{c}^{n}\left(V_{\lambda}\right)^{G_{\max }}$ equals the order of the Picard-Fuchs equation for $X_{\lambda}$.

Proof. Since $V_{\lambda}$ is smooth we have by Poincaré duality [3] that

$$
\operatorname{dim} H_{c}^{n}\left(V_{\lambda}\right)^{G_{\max }}=\operatorname{dim} H^{n}\left(V_{\lambda}\right)^{G_{\max }} .
$$

We now calculate the latter dimension. The group $G_{\max }$ consists of the $\left(g_{1}, \ldots, g_{n}\right)$ in $(\mathbf{Z} / d \mathbf{Z})^{n}$ such that

$$
\sum_{i=1}^{n} g_{i} b_{i} \equiv 0 \bmod d .
$$


From [16, Lemma 4.2] it follows that $G_{\max }$ fixes the differential form $\omega_{\mathbf{k}}$ if and only if $\mathbf{k} \equiv t \mathbf{b}$ $\bmod d$ for some $t \in \mathbf{Z} / d \mathbf{Z}$. Hence $H^{n}\left(V_{\lambda}\right)^{G_{\max }}$ is spanned by $\omega_{t \mathbf{b}}$ where $t \in\{0,1, \ldots, d-1\}$ such $t \mathbf{b} \bmod d$ has no zero entry.

The number of $t \in \mathbf{Z} / d \mathbf{Z}$ such that $t \mathbf{b} \bmod d$ has a zero entry equals the number of $t \in$ $\{0, \ldots, d-1\}$ for which there exists an $i$ and an integer $k$ such that $t b_{i}=k d$, or, equivalently,

$$
\frac{t}{d}=\frac{k}{b_{i}}
$$

Since $0 \leq t<d$ we may assume that $0 \leq k<b_{i}$. Using the notation from [8, Section 2] we have that the elements on the left hand side are in the set they call $\alpha$ and the elements on the right hand side are in the set $\beta$. In particular, the number of $t$ such that $t \mathbf{b}$ has no zero entry equals $d-\# \alpha \cap \beta$. Gährs [10, Theorem 2.8] showed that this number equals the order of the Picard-Fuchs equation.

Proposition 2.24. Suppose $w_{0}=\cdots=w_{n}=1, d=n+1$ and $a_{i}=1$ for $i=0, \ldots, n$. Then the factor $R_{\lambda}^{\prime}$ found in [8] is the characteristic polynomial of Frobenius acting on $H^{n}\left(V_{\lambda}\right)^{G_{\max }}$. In particular, $R_{\lambda}^{\prime} \in \mathbf{Q}[T]$ and $R_{\lambda}=R_{\lambda}^{\prime}$.

Proof. Since $\mathbf{P}(\mathbf{w})=\mathbf{P}^{n}$ we have $U_{\lambda}=\tilde{U}_{\lambda}$. Hence we can discuss differential forms on the complement of $X_{\lambda}$.

The factor $R_{\lambda}^{\prime}(T)$ obtained in [8] using the $p$-adic Picard-Fuchs equation in Dwork cohomology. The main result from [12] yields a differential equation satisfied by the Frobenius operator on $H_{\mathrm{MW}}^{n}\left(U_{\lambda}, \mathbf{Q}_{q}\right)$ and that this differential equation can also be found using Dwork cohomology. In particular, $R_{\lambda}^{\prime}(T)$ is the characteristic polynomial of Frobenius acting on the subspace $P$ containing $\omega_{\mathbf{a}}$ and invariant under the Picard-Fuchs operator. This subspace $P$ is contained in the span of $\left\{\omega_{s \mathbf{a}}: s=1,2, \ldots\right\}$.

Pick a form $\tilde{\omega}_{t a}$ restrict this form to $U_{\lambda}^{*}$ and then pull it back to a form on $V_{\lambda}^{*}$. Then this pull back is $\omega_{t \mathbf{b}}$. This form is defined on all of $V_{\lambda}$. Hence the pullback of $P$ to $H^{n}\left(V_{\lambda}\right)$ is well-defined and is contained in $H^{n}\left(V_{\lambda}\right)^{G_{\max }}$. Hence $P$ is a subspace of $H^{n}\left(V_{\lambda}\right)^{G_{\max }}$. Since both spaces have the same dimension by Lemma 2.23 they coincide, i.e., $P \cong H^{n}\left(V_{\lambda}\right)^{G_{\max }}$ as vector spaces with Frobenius action.

Now $R_{\lambda}$ is the characteristic polynomial of $q^{n}$ Frob $^{-1}$ acting on $P$. Using Poincaré duality this equals the characteristic polynomial of Frobenius acting on $H_{c}^{n}\left(V_{\lambda}\right)^{G_{\max }}$. This yields the first claim. The obtained polynomial is in $\mathbf{Q}[T]$ by [16, Lemma 4.3] and hence $R_{\lambda}^{\prime}(T)=R_{\lambda}(T)$.

\section{Case of quartic surfaces}

In this section we consider the case of invertible quartic polynomials. Up to permutation of the coordinates there are 10 invertible quartic polynomials in four variables. For each of these quartics we take $\mathbf{a}=(1,1,1,1)$ as the deformation vector.

In Fig. 1 we list the 10 families, which we denote here with $X_{\lambda}^{(i)}$. We provide the following information in the table. In the column " $d$ " we list the minimal degree of a Fermat cover of the central fiber. When we discuss one of the examples we always assume that $\operatorname{gcd}(q, d)=1$. In the next column we list the deformation vector $\mathbf{b}:=(1,1,1,1)^{\mathrm{T}} B$. Hence the corresponding Fermat cover $Y_{\lambda}^{(i)}$ is defined by

$$
x_{0}^{d}+x_{1}^{d}+x_{2}^{d}+x_{3}^{d}+\lambda x_{0}^{b_{0}} x_{1}^{b_{1}} x_{2}^{b_{2}} x_{3}^{b_{3}} .
$$

Let $G$ be the Galois group of the function field extension corresponding to the morphism $Y_{\lambda}^{*} \rightarrow X_{\lambda}^{*}$. The next two columns deal with $H^{3}\left(V_{\lambda}\right)^{G}$, for $\lambda$ such that $Y_{\lambda}$ is smooth. In the 


\begin{tabular}{|c|c|c|c|c|c|c|}
\hline$i$ & $F_{0}$ & $d$ & $(1,1,1,1)^{\mathrm{T}} B$ & $P F$ & $\operatorname{dim} W_{\lambda}$ & $c$ \\
\hline 1 & $x_{0}^{4}+x_{1}^{4}+x_{2}^{4}+x_{3}^{4}$ & 4 & $(1,1,1,1)$ & 3 & 18 & 0 \\
2 & $x_{0}^{4}+x_{1}^{4}+x_{2} x_{3}\left(x_{2}^{2}+x_{3}^{2}\right)$ & 8 & $(2,2,2,2)$ & 3 & 12 & 6 \\
3 & $x_{0} x_{1}\left(x_{0}^{2}+x_{1}^{2}\right)+x_{2} x_{3}\left(x_{2}^{2}+x_{3}^{2}\right)$ & 8 & $(2,2,2,2)$ & 3 & 10 & 8 \\
4 & $x_{0}^{4}+x_{1} x_{2}^{3}+x_{2} x_{3}^{3}+x_{3} x_{1}^{3}$, & 28 & $(7,7,7,7)$ & 3 & 18 & 0 \\
5 & $x_{0} x_{1}^{3}+x_{1} x_{2}^{3}+x_{2} x_{3}^{3}+x_{3} x_{0}^{3}$ & 80 & $(20,20,20,20)$ & 3 & 16 & 2 \\
6 & $x_{0}^{4}+x_{1}^{4}+x_{2}^{3} x_{3}+x_{3}^{4}$ & 12 & $(3,3,4,2)$ & 6 & 12 & 3 \\
7 & $x_{0} x_{1}\left(x_{0}^{2}+x_{1}^{2}\right)+x_{2}^{3} x_{3}+x_{3}^{4}$ & 24 & $(6,6,8,4)$ & 6 & 8 & 7 \\
8 & $x_{0}^{3} x_{1}+x_{1}^{4}+x_{2}^{3} x_{3}+x_{3}^{4}$ & 12 & $(4,2,4,2)$ & 4 & 12 & 5 \\
9 & $x_{0}^{4}+x_{1}^{3} x_{2}+x_{2}^{3} x_{3}+x_{3}^{4}$ & 36 & $(9,12,8,7)$ & 18 & 0 & 3 \\
10 & $x_{0}^{3} x_{1}+x_{1}^{3} x_{2}+x_{2}^{3} x_{3}+x_{3}^{4}$ & 108 & $(36,24,28,20)$ & 18 & 0 & 3 \\
\hline
\end{tabular}

Figure 1. The 10 families $X_{\lambda}^{(i)}$.

column $P F$ we list the dimension of $H^{3}\left(V_{\lambda}\right)^{G_{\max }}$. We calculated this entry as follows: from the results from [16, Section 4] it follows that a basis for this vector space consists of those $\omega_{\mathbf{k}}$ such that all entries of $\mathbf{k}$ are between 1 and $d-1$ and there is a $t \in \mathbf{Z}$ such that $\mathbf{k} \equiv t \mathbf{b} \bmod d$. It is straight forward to determine the number of these $\mathbf{k}$. As discussed in the previous section, this number equals the order of the Picard-Fuchs equation of $X_{\lambda}^{i}$.

The next column concerns the subspace $W_{\lambda}^{(i)} \subset H^{3}\left(V_{\lambda}^{(i)}\right)^{G}$. The $\omega_{\mathbf{k}}$ such that each of the entries of $\mathbf{k}$ is in $\{1, \ldots, d-1\}$ and there exists a vector $\mathbf{m} \in \mathbf{Z}^{4}$ such that $\mathbf{k} \equiv \mathbf{m} A \bmod d$ form a basis for $H^{3}\left(V_{\lambda}^{(i)}\right)^{G}$. For each of the 10 examples we checked for each $\mathbf{k}$ if such a $\mathbf{m}$ existed or not and used this to calculate $\operatorname{dim} W_{\lambda}^{(i)}=\operatorname{dim} H^{3}\left(V_{\lambda}^{(i)}\right)^{G}-\operatorname{dim} H^{3}\left(V_{\lambda}^{(i)}\right)^{G_{\max }}$.

For the families 1, 2, 3, 6, 7 we listed all these $\mathbf{k}$ in Fig. 2 (they are enlisted in the corresponding column in Fig. 2, in the first column there is a choice for a possible $\mathbf{m}$, the forms marked with $(P F)$ are in $\left.H^{3}\left(V_{\lambda}\right)^{G_{\max }}\right)$. For $i=4,5,8,9,10$ we will describe $W_{\lambda}^{(i)}$ in the examples below.

Finally, the column $c$ then equals $21-\operatorname{dim} H^{3}\left(V_{\lambda}\right)^{G}$. As we argued in the introduction the subspace $C$ of dimension $c$ and $W_{\lambda}^{(i)}$ are generated by classes of curves on $X_{\lambda}^{(i)}$. For the families $i=1,2,3,6,7,9,10$ we give a recipe to find linear combinations of curves on $X_{\lambda}^{(i)}$, which generate $C$ and $W_{\lambda}^{(i)}$. In fact, for all $i$ we have that $C$ is generated by curves, each of which is contained in one of the coordinate hyperplanes. These curves are easy to find for each $i$. For $i=9,10$ we have that $W_{\lambda}^{(i)}=0$. For $i=1,2,3,6,7$ we can find various del Pezzo surfaces of degree 2 together with morphisms of degree 2, such that linear combinations of pull backs of curves from these del Pezzo surfaces generated $W_{\lambda}^{(i)}$. For $i=5$ we have a similar procedure using del Pezzo surfaces of degree 5.

The first five families have a single common cover, also the sixth and seventh family have a common cover. The common factor of the first five examples has degree 3 . However, the first three examples have a common factor of degree 5 and the first and the second example have a common factor of degree 7 .

The following proposition now shows that claim about $W_{\lambda}^{(i)}$ for $i=1,2,3,6,7$ :

Proposition 3.1. Consider one of the families $X_{\lambda}^{(i)}$ with $i \in\{1,2,3,6,7\}$ from Fig. 1. Then there exist families of del Pezzo surfaces $S_{\lambda}^{(i, j)}$ and degree 2 morphisms $\varphi_{\lambda}^{(i, j)}: X_{\lambda}^{(i)} \rightarrow S_{\lambda}^{(i, j)}$ such that if $i \in\{1,6,7\}$ then for almost all $\lambda$ we have that

$$
W_{\lambda}^{(i)} \subset \sum_{j} \varphi_{\lambda}^{(i, j) *}\left(H^{2}\left(S_{\lambda}^{(i, j)}\right)\right)
$$




\begin{tabular}{|c|c|c|c|c|c|}
\hline $\mathbf{m}$ & 3.1 & 3.2 & 3.3 & 3.6 & 3.7 \\
& & & & $A=10, B=11$ & \\
\hline 1111 & $1111(P F)$ & $2222(P F)$ & $2222(P F)$ & $3342(P F)$ & $6,6,8,4(P F)$ \\
1124 & - & - & - & $338 A(P F)$ & $6,6,16,20(P F)$ \\
1133 & 1133 & 2266 & 2266 & - & - \\
1214 & - & - & - & $364 B$ & $3,15,8,22$ \\
1223 & 1223 & 2437 & 1537 & 3687 & $3,15,16,14$ \\
1232 & 1232 & 2473 & 1573 & - & - \\
1313 & 1313 & - & - & 3948 & - \\
1322 & 1322 & 2644 & - & 3984 & - \\
1331 & 1331 & - & - & - & - \\
2114 & - & - & - & $634 B$ & $15,3,8,22$ \\
2123 & 2123 & 4237 & 5137 & 6387 & - \\
2132 & 2132 & 4273 & 5173 & - & $15,3,16,14$ \\
2222 & $2222(P F)$ & $4444(P F)$ & $4444(P F)$ & $6684(P F)$ & $12,12,16,8(P F)$ \\
2213 & 2213 & - & - & $6648(P F)$ & $12,12,8,16(P F)$ \\
2231 & 2231 & - & - & - & - \\
2312 & 2312 & 4615 & 3715 & 6945 & $9,21,8,10$ \\
2321 & 2321 & 4651 & 3751 & 6981 & $9,21,16,2$ \\
3113 & 3113 & - & - & 9348 & - \\
3122 & 3122 & 6244 & - & 9384 & - \\
3131 & 3131 & - & - & - & - \\
3212 & 3212 & 6415 & 7315 & 9645 & $21,9,8,10$ \\
3221 & 3221 & 6451 & 7351 & 9681 & $21,9,16,2$ \\
3311 & 3311 & 6622 & 6622 & $9942(P F)$ & $18,18,8,4(P F)$ \\
3324 & - & - & - & $998 A(P F)$ & $18,18,16,20(P F)$ \\
3333 & $3333(P F)$ & $6666(P F)$ & $6666(P F)$ & - & - \\
\hline & & & & & \\
\hline
\end{tabular}

Figure 2. Generators for $H^{3}\left(V_{\lambda}^{(i)}\right)^{G}$.

and if $i \in\{2,3\}$ then for almost all $\lambda$ we have that

$$
W_{\lambda}^{(i)} \cap \sum_{j} \varphi_{\lambda}^{(i, j) *}\left(H^{2}\left(S_{\lambda}^{(i, j)}\right)\right)
$$

has codimension 2 in $W_{\lambda}^{(i)}$ and the forms $\tilde{\omega}_{1133}$, $\tilde{\omega}_{3311}$ generate a complementary subspace in $W_{\lambda}^{(i)}$.

If $i=3$ or $i=7$ or $q \equiv 1 \bmod 4$ then we can take the $S_{\lambda}^{(i, j)}$ to be defined over $\mathbf{F}_{q}$. If $q \equiv 3 \bmod 4$ and $i \in\{1,2,6\}$ then some of the $S_{\lambda}^{(i, j)}$ are only defined over $\mathbf{F}_{q^{2}}$.

Proof. Note that $W_{\lambda}^{(i)}$ is spanned by $\tilde{\omega}_{\mathbf{m}}$ where $\mathbf{m}$ are precisely these entries from the first column of Fig. 2 such that in the column corresponding to $i$ there the entry is different from "-" and is without the mark " $(P F)$ ". Note also that in the notation of the previous section we have $\tilde{U}_{\lambda}^{(i)}=U_{\lambda}^{(i)}$. Hence we denote differential forms on the complement of $X_{\lambda}^{(i)}$ with $\tilde{\omega}$ and forms on the complement of $Y_{\lambda}^{(i)}$ with $\omega$.

A defining polynomial for $X_{0}^{(i)}$ can be found in Fig. 1. Recall that for each family we took $(1,1,1,1)$ as the deformation factor. In particular, each of the five families under consideration is each invariant under the automorphisms $\sigma$ and $\tau$ defined by

$$
\sigma\left(x_{0}, x_{1}, x_{2}, x_{3}\right):=\left(x_{1}, x_{0}, x_{2}, x_{3}\right), \quad \tau\left(x_{0}, x_{1}, x_{2}, x_{3}\right):=\left(-x_{1},-x_{0}, x_{2}, x_{3}\right) .
$$


A straightforward calculation shows that the quotients of $X_{\lambda}^{(i)}$ by $\sigma$ and by $\tau$ are both surfaces of degree 4 in $\mathbf{P}(1,1,1,2)$, and that for general $\lambda$ they are smooth (explicit equations for these surfaces can be found in the appendix). Hence the quotient surfaces are del Pezzo surfaces of degree 2. Denote the corresponding surfaces with $S_{\lambda}^{(i, 1)}$ and $S_{\lambda}^{(i, 2)}$

Let $\mathbf{m}=(a, b, c, d)$ with $a, b, c, d \in\{1,2,3,4\}$ be such that $a+b+c+d \equiv 0 \bmod 4$. Then $\tilde{\omega}_{\mathbf{m}}+\sigma^{*} \tilde{\omega}_{\mathbf{m}}$ is invariant under $\sigma^{*}$ and therefore contained in $\pi_{1}^{*}\left(H^{2}\left(S_{\lambda}^{(i, 1)}\right)\right)$. Similarly, $\tilde{\omega}_{\mathbf{a}}+\tau^{*} \tilde{\omega}_{\mathbf{a}}$ is contained in $\pi_{2}^{*}\left(H^{2}\left(S_{\lambda}^{(i, 2)}\right)\right)$. Now $\tilde{\omega}_{\mathbf{m}}+\sigma^{*} \tilde{\omega}_{\mathbf{m}}=\tilde{\omega}_{a b c d}-\tilde{\omega}_{b a c d}$ and $\tilde{\omega}_{\mathbf{m}}+\tau^{*} \tilde{\omega}_{\mathbf{m}}=\omega_{a b c d}+$ $(-1)^{a+b+1} \omega_{\text {bacd }}$. Hence, if $a+b$ is odd then $\tilde{\omega}_{\mathbf{m}} \in \pi_{1}^{*}\left(H^{2}\left(S_{\lambda}^{(i, 1)}\right)\right)+\pi_{2}^{*}\left(H^{2}\left(S_{\lambda}^{(i, 2)}\right)\right)$. In the case $i=7$ we have that $W_{\lambda}^{(i)}$ is generated by forms $\tilde{\omega}_{\mathbf{m}}$ with $a+b$ odd and we finished this case. In the case $i=3$ we have that $W_{\lambda}^{(i)}$ is generated by forms with $a+b$ odd and the two forms $\tilde{\omega}_{1133}$ and $\tilde{\omega}_{3311}$. Hence we finishes also this case.

In the remaining cases $i=1,2,6$ we have a further automorphism

$$
\tau_{1}:\left(x_{0}, x_{1}, x_{2}, x_{3}\right) \mapsto\left(I x_{1},-I x_{0}, x_{2}, x_{3}\right),
$$

where $I^{2}=-1$. Denote $S_{\lambda}^{3, i}$ the quotient by $\tau_{1}$. If $q \equiv 1 \bmod 4$ then $S_{\lambda}^{3, i}$ is defined over $\mathbf{F}_{q}$, but if $q \equiv 3 \bmod 4$ then it is only defined over $\mathbf{F}_{q^{2}}$.

Note that

$$
\tau_{1}^{*}\left(\tilde{\omega}_{a b c d}\right)=(-1)^{b+1}(I)^{a+b} \tilde{\omega}_{b a c d} .
$$

Hence if $b$ is odd and $a+b \equiv 0 \bmod 4$ then $\tilde{\omega}_{a b c d}+\tilde{\omega}_{b a c d}$ is fixed under $\tau_{1}^{*}$ and, as above, we find that $\tilde{\omega}_{a b c d}$ is in $\pi_{1}^{*}\left(H^{2}\left(S_{\lambda}^{i, 1}\right)\right)+\pi_{3}^{*}\left(H^{2}\left(S_{\lambda}^{i, 3}\right)\right)$.

Using Fig. 2 we can conclude that we recovered any $\tilde{\omega}_{\mathbf{m}}$ such that the first two entries are distinct. This finishes the proof for the case $i=6$. In the case $i=2$, we only miss the forms $\tilde{\omega}_{1133}$ and $\tilde{\omega}_{3311}$, hence we are also done in this case. In the case $i=1$ there is a $S_{4}$ symmetry we can use. We recover all $\tilde{\omega}_{\mathbf{k}}$ with at least two distinct entries in $\mathbf{k}$ and this finishes also this case.

Remark 3.2. In the cases $i=2,3$ we do not recover $\tilde{\omega}_{1133}$ and $\tilde{\omega}_{3311}$. However, the families $i=1,2,3$ have

$$
x_{0}^{24}+x_{1}^{24}+x_{2}^{24}+x_{3}^{24}+\lambda\left(x_{0} x_{1} x_{2} x_{3}\right)^{6}
$$

as a common cover. For each of three families the form $\tilde{\omega}_{1133}$ is pulled back to the form $\omega_{6,6,18,18}$ on $V_{\lambda}$. Hence we can use $X_{\lambda}^{(1)}$ to express this form in terms of divisors pulled back form $S_{\lambda}^{1,3}$.

In the following examples we discuss how to find generators for the subspace $C$. For the examples $i=4,5,8$ we list a basis for $H^{3}\left(V_{\lambda}^{(i)}\right)^{G_{\max }}$ and also discuss strategies to find generators for $W_{\lambda}$.

Example 3.3. For the case $i=1,2,3,6,7$ we note that Proposition 3.1 yields a basis for $W_{\lambda}^{(i)}$ in terms of curves pulled back from del Pezzo surfaces $S^{(i, j)}$. In the appendix we will explain how to find these curves.

For each of these cases we can find generators for $C$ in each of these cases, but the approach depends on $i$ :

$i=1 C=0$ in this case.

$i=2$ The curves given by $x_{3}=0, x_{0}=-I^{k} x_{1}$ and the ones given by $x_{4}=0, x_{0}=-I^{k} x_{1}$ are in $C$, with $I^{2}=-1$. One easily checks that they generate $C$. These curves can also be obtained by pulling back curves from the del Pezzo quotients: For example, consider the quotient by the automorphism $\sigma:\left(x_{0}, x_{1}, x_{2}, x_{3}\right) \mapsto\left(x_{1}, x_{0}, x_{2}, x_{3}\right)$. We find that 
$\omega_{a b c d}-\omega_{b a c d}$ is a 1 eigenvector if $(a, b) \neq(b, a)$. However, there are only 5 such eigenvectors. The Picard group of the del Pezzo surface has rank 8. The additional three divisors are the hyperplane class and the two curves pulled back from the curves $x_{3}=x_{1}^{2}+x_{2}^{2}=0$ and $x_{4}=x_{1}^{2}+x_{2}^{2}=0$.

$i=3$ We have that for $j<2$ and $k>1$ the line $x_{j}=x_{k}=0$ is contained in $X_{\lambda}^{(3)}$ as are $x_{m}=0$, $x_{2}= \pm I x_{3}, m \leq 2$ and $x_{m}=0, x_{1}= \pm I x_{0}, m \in\{2,3\}$. These are 12 curves, but generate a rank 9 sublattice of the Picard lattice, and this lattice contains the hyperplane class. Linear combinations of these curves span $C$.

$i=6$ As in the case $i=2$ in this case we have that the automorphism $\sigma$ fixes only six eigenvectors of the form $\tilde{\omega}_{\mathbf{k}}-\tilde{\omega}_{\sigma(\mathbf{k})}$. The seventh eigenvector is the class of the curve $x_{3}=0, x_{0}^{2}+x_{1}^{2}$, which is an element of $C$. The other coordinate hyperplanes yields three further curves, contributing another two to the Picard number.

$i=7$ In this case we take $x_{0}=0$ or $x_{1}=0$ then we find $x_{3}\left(x_{2}^{3}+x_{3}^{3}\right)=0$. In this way we find 8 lines, contributing six to cohomology.

We discuss now the other five examples:

Example 3.4. Consider now the case $i=4$. In this case the Fermat cover $Y_{\lambda}^{(4)}$ has degree 28. Let $G$ be the associated group of torus automorphisms. Then the multiples $\omega_{\mathbf{k}}$ with $\mathbf{k}$ a multiple of $(7,7,7,7)$ generate a rank 3 subspace of $H^{3}\left(V_{\lambda}^{(4)}\right)^{G}$ which is common to the examples $i=$ $1,2,3$. The other monomial types associated with forms in $H^{3}\left(V_{\lambda}^{(4)}\right)^{G}$ are

$$
(7,11,15,23),(7,3,27,19),(14,2,18,22),(14,6,26,10),(21,1,9,25),(21,5,17,13)
$$

and those obtained by a cyclic permutation of the last three coordinates. In particular, these $\omega_{\mathbf{k}}$ generate already a rank 21 subspace of $H^{3}\left(V_{\lambda}\right)^{G}$, which has dimension at most 21 . Hence we found a basis for $H^{3}\left(V_{\lambda}^{(4)}\right)^{G}$ and we have $C=0$ in this case.

One can obtain some information on the zeta function as follows. If $q \equiv 1 \bmod 28$ then we can factor the zeta function over $\mathbf{Q}_{q}$ according to strong equivalence classes (cf. [16, Section 4]). The strong equivalence class of $(7,7,7,7)$ consists further of $(14,14,14,14)$ and $(21,21,21,12)$. The class of $(7,11,23,15)$ consists further of $(14,18,2,22)$ and of $(21,25,9,1)$. The class of $(7,3,19,27)$ consists further of $(14,10,26,6)$ and of $(21,17,5,13)$. The other classes can be obtained by permutation the last three coordinates. In particular, we find that the characteristic polynomial on $H^{3}\left(V_{\lambda}^{(4)}\right)^{G}$ can be written as $P_{3} Q_{3}^{3} R_{3}^{3}$, where $P_{3}, Q_{3}$ and $R_{3}$ are in $\mathbf{Q}_{q}[T]$ and have degree 3 . The polynomial $P_{3}$ is the common factor and by Corollary 2.21 in $\mathbf{Q}[T]$. Since $P_{3} Q_{3}^{3} R_{3}^{3} \in \mathbf{Q}[T]$ we find that also $Q_{3}(T) R_{3}(T)$ is in $\mathbf{Q}[T]$.

The results from [15, Section 5] yield three explicit matrices, each $3 \times 3$, whose entries are rational functions of generalised $p$-adic hypergeometric functions, such that the three corresponding characteristic polynomials are $P_{3}, Q_{3}$ and $R_{3}$.

As mentioned in the introduction of this paper, we did not find a complete set of generators for generic Picard group for two of the ten families. This family is one of these two families.

Example 3.5. The degree of the Fermat cover of the fifth example is 80 . The monomial type $(20,20,20,20)$ and its two multiplies in $H^{3}\left(V_{\lambda}^{(5)}\right)$ yield the factor common with the examples $i=1,2,3,4$.

The other monomial types associated with classes in $H^{3}\left(V_{\lambda}^{(5)}\right)^{G}$, are

$$
(4,52,36,58),(24,72,56,8),(44,12,76,28),(64,32,16,48)
$$

and the cyclic permutations of these. Hence $W_{\lambda}^{(5)}$ has dimension 16 . The subspace $C$ has dimension 2 and contains the classes of the lines $x_{0}=x_{2}=0$ and $x_{1}=x_{3}=0$. 
To find the curves contributing to the rank 16 part, we can use permutations, similarly as in the above examples. The cyclic permutation of $1,2,3,4$ is odd. Denote this permutation by $\sigma_{0}$. The quotient by this permutation is a del Pezzo surface of degree 5 .

Fix now a primitive fifth root of unity $\zeta$. Let

$$
\rho:=\left(x_{0}, x_{1}, x_{2}, x_{3}\right) \mapsto\left(\zeta x_{0}, \zeta^{3} x_{1}, \zeta^{4} x_{2}, \zeta^{2} x_{1}\right)
$$

For $i=1,2,3,4$ we set $\sigma_{i}=\sigma_{0} \rho^{i}$. Then each $\sigma_{i}$ has order 4 .

Let $\mathbf{m}$ be a monomial types such that $\tilde{\omega}_{\mathbf{m}}$ is pulled back to one of the 16 forms $H^{3}\left(V_{\lambda}^{(5)}\right)^{G}$ not a multiple of $(20,20,20,20)$.

Consider now $\left\{\sum_{j=0}^{3} \sigma_{i}^{j} \tilde{\omega}_{\mathbf{m}}: i=0, \ldots, 3\right\}$. A direct calculation using a Vandermonde determinant shows that these four forms are linearly independent and that their span contains $\tilde{\omega}_{\mathbf{k}}$. Hence $\tilde{\omega}_{\mathbf{k}}$ is contained in the subspace spanned by $\bigcup_{i=1}^{4} H^{3}\left(U_{\lambda}\right)^{\sigma_{i}}$. So each of the $\tilde{\omega}_{\mathbf{k}}$ can be expressed as a linear combination of curves on the del Pezzo surface $X_{\lambda}^{(5)} / \sigma_{i}$, with $i=0,1,2,3$.

Using the terminology of [15, Section 6] we have two weak equivalence classes of monomial types, one consisting of three monomial types and consisting of 16 monomial types. The large class decomposes in four strong equivalence classes. These four strong equivalence classes are in one $\sigma$-orbit. From this we obtain if $q \equiv 1 \bmod 20$ then the characteristic polynomial of Frobenius is $P_{4} Q_{4}^{4}$, where both $P_{4}$ and $Q_{4}$ are of degree 4 .

A different approach to find curves on $X_{\lambda}^{(5)}$ would be to use the line $x_{0}=x_{2}=0$ to find an elliptic fibration. A Weierstrass equation for this fibration is

$$
y^{2}=x^{3}-27 s^{4}\left(\lambda^{4}+144\right) x-54 s\left(-s^{5} \lambda^{6}+864 s^{10}+648 s^{5} \lambda^{2}+864\right) .
$$

For general $\lambda$ the fibration has 2 fibers of type $I I$ and 20 fibers of type $I_{1}$. The sections of this fibration and the fiber class generate the Picard group for general $\lambda$.

Example 3.6. For $i=8$ that the Fermat cover has degree 12, and the deformation vector is pulled back to 4242 . The Picard-Fuchs equation has order 4 . The other monomial types $\omega_{\mathbf{k}}$ are built up from pairs from $42,45,48,4 B, 81,84,87,8 A$ (where $A=10, B=11$ ) such that the entries add up to a multiple of 12 and such that $\mathbf{k}$ is not a multiple of 4242 . In total we find 12 such forms.

The complementary five-dimensional subspace comes from coordinate plane sections, i.e., $x_{1}=0$ yields $x_{3}\left(x_{2}^{3}+x_{3}^{3}\right)$, and also $x_{3}=0$ contributes. The total contribution is 5 . We do not have any odd permutation to work with. However, this surface has many elliptic fibrations and one may be able to work with them.

As mentioned in the introduction of this paper, we did not find a complete set of generators for generic Picard group for two of the ten families. This family is one of these two families.

Example 3.7. In the ninth example we have that the common cover has degree 36 . The deformation monomial has exponents $9,12,8,7$. There are 18 multiples of this vector without a zero in $\mathbf{Z} / 36 \mathbf{Z}$. Hence the Picard-Fuchs equation has degree 18. Moreover, the curves $x_{2}=0$, $x_{0}=i^{k} x_{4}$ together with the hyperplane class generate the generic Picard group.

Example 3.8. In the tenth examples we have that the Fermat cover has degree 108. The deformation monomial has exponents 36, 24, 28, 20. There are 18 multiples of this vector without a zero in $\mathbf{Z} / 108 \mathbf{Z}$. Hence the Picard-Fuchs equation has degree 18. Moreover, we have the curves $x_{1}=x_{3}=0, x_{1}=0, x_{2}=\omega^{i} x_{3}$ and $x_{3}=0, x_{0}^{3}-x_{1}^{2} x_{2}=0$. This are five curves admitting two relations. 
Remark 3.9. In two cases we did not find generators. In these two cases different there is no permutation $\sigma$ of the coordinates which is automorphism of the family and such that the quotient surface is a rational surface. In the other examples with nontrivially $W_{\lambda}$, this space was generated by pull backs of curves coming from rational surfaces.

It is the author's experience that in characteristic zero, establishing explicit curves generating the Picard group of a surface, is an easier problem when working with surfaces with $h^{2,0}=0$ then when working with surfaces with $h^{2,0}>0$. This can be partly explained by the fact that degrees and intersection numbers of generators of the Picard group are determined by the topology of the surface in the case $h^{2,0}=0$, but not in the case $h^{2,0}>0$.

A similar problem is determining a basis of the Mordell-Weil group of an elliptic K3 surfaces (which is equivalent to determining generators for the Nèron-Severi group of that surface). This turned out to be much simplified if the $K 3$ surface is in various ways the pull back of a rational elliptic surface. (E.g., see $[7,14,19]$.)

\section{A Bitangents to special plane quartics}

In Section 3 we considered ten pencils of quartic surfaces. In Proposition 3.1 we showed that five of these pencils are (each in multiple ways) double covers of pencils of del Pezzo surfaces of degree two and we showed how the knowledge of the Picard group of these del Pezzo surfaces is sufficient to determine the generic Picard group of each pencil. In this section we explain how one can find explicit generators for the Picard group of these del Pezzo surfaces. It is well-known that such a surface is a double cover of $\mathbf{P}^{2}$ ramified along a quartic curve.

If the quartic curve is smooth then its has 28 bitangents. These bitangents are pulled back to two lines on the del Pezzo surface, and these lines generate the Picard group.

In order to find explicit equations for the del Pezzo surfaces of degree 2 and the quartic curves we are going to make the steps from the proof of Proposition 3.1 explicit. This proposition applies only to $X_{\lambda}^{(i)}$ with $i \in\{1,2,3,6,7\}$, hence we concentrate on these cases. To ease the calculations

we start by decomposing the defining polynomials for $X_{\lambda}^{(i)}$ in sums of two polynomials. Therefore define the following polynomials

$$
\begin{aligned}
& f_{1}\left(x_{0}, x_{1}, x_{2}, x_{3}\right):=x_{0}^{4}+x_{1}^{4}+\lambda x_{0} x_{1} x_{2} x_{3}, \\
& f_{2}\left(x_{0}, x_{1}, x_{2}, x_{3}\right):=x_{0} x_{1}\left(x_{0}^{2}+x_{1}^{2}\right)+\lambda x_{0} x_{1} x_{2} x_{3}, \\
& g_{1}\left(x_{2}, x_{3}\right):=x_{2}^{4}+x_{3}^{4}, \\
& g_{2}\left(x_{2}, x_{3}\right):=x_{2} x_{3}\left(x_{2}^{2}+x_{3}^{2}\right), \\
& g_{3}\left(x_{2}, x_{3}\right):=x_{2}^{3} x_{3}+x_{3}^{4}, \\
& h_{1}\left(u, v, x_{2}, x_{3}\right):=u^{4}-4 u^{2} v+2 v^{2}+\lambda v x_{2} x_{3}, \\
& h_{2}\left(u, v, x_{2}, x_{3}\right):=v\left(u^{2}-2 v\right)+\lambda v x_{2} x_{3} .
\end{aligned}
$$

The five pencils of quartic surfaces under consideration are defined by the vanishing of

$$
f_{1}+g_{1}, \quad f_{1}+g_{2}, \quad f_{2}+g_{2}, \quad f_{1}+g_{3}, \quad f_{2}+g_{3} .
$$

\section{A.1 $S_{\lambda}^{(i, 1)}$}

As we noted in the proof of Proposition 3.1 each of these families is invariant under the automorphism $\sigma:\left(x_{0}, x_{1}, x_{2}, x_{3}\right) \mapsto\left(x_{1}, x_{0}, x_{2}, x_{3}\right)$.

In particular, each of the defining polynomials is also a polynomial in $x_{0}+x_{1}, x_{0} x_{1}, x_{2}, x_{3}$. We defined $h_{1}, h_{2}$ such that

$$
h_{j}\left(x_{0}+x_{1}, x_{0} x_{1}, x_{2}, x_{3}\right)=f_{j}\left(x_{0}, x_{1}, x_{2}, x_{3}\right) .
$$


Therefore the quotient $S_{\lambda}^{(i, 1)}$ of $X_{\lambda}^{(i)}$ by $\sigma$ is the zeroset of

$$
h_{1}+g_{1}, \quad h_{1}+g_{2}, \quad h_{2}+g_{2}, \quad h_{1}+g_{3}, \quad h_{2}+g_{3}
$$

in $\mathbf{P}(1,2,1,1)$. These polynomials define five families of surfaces in $\mathbf{P}(1,2,1,1)$. The general member is a del Pezzo surface of degree 2. The rational map $\mathbf{P}(1,2,1,1) \rightarrow \mathbf{P}^{2}$ defined by $\left(u: v: x_{2}: x_{3}\right) \rightarrow\left(u: x_{2}: x_{3}\right)$ is defined on all of $S_{\lambda}^{(i, 1)}$. It establishes this surfaces as a double cover of $\mathbf{P}^{2}$ ramified along the zeroset of $q_{i}$, the discriminant $q_{i}$ of the defining polynomial of $S_{\lambda}^{(i, 1)}$ considered as polynomial in $v$. These discriminant are straightforward to compute. We list them here:

$$
\begin{aligned}
& q_{1}:=-8 x_{2}^{4}+\lambda^{2} x_{2}^{2} x_{3}^{2}-8 \lambda x_{2} x_{3} u^{2}-8 x_{3}^{4}+8 u^{4}, \\
& q_{2}:=-8 x_{2}^{3} x_{3}+\lambda^{2} x_{2}^{2} x_{3}^{2}-8 x_{2} x_{3}^{3}-8 \lambda x_{2} x_{3} u^{2}+8 u^{4}, \\
& q_{3}:=8 x_{2}^{3} x_{3}+\lambda^{2} x_{2}^{2} x_{3}^{2} t+8 x_{2} x_{3}^{3}+2 \lambda x_{2} x_{3} u^{2}+u^{4}, \\
& q_{6}:=-8 x_{2}^{4}+\lambda^{2} x_{2}^{2} x_{3}^{2}-8 x_{2}^{3} x_{3}-8 \lambda x_{2} x_{3} u^{2}+8 u^{4}, \\
& q_{7}:=8 x_{2}^{4}+\lambda^{2} x_{2}^{2} x_{3}^{2}+8 x_{2}^{3} x_{3}+2 \lambda x_{2} x_{3} u^{2}+u^{4} .
\end{aligned}
$$

Our aim is to find the bitangents to these curves and then pull them back to $X_{\lambda}^{(i)}$. If $\lambda$ is chosen such that the quartic curve is smooth then there are 28 bitangents. We start by looking for bitangents of the shape $u=a_{2} x_{2}+a_{3} x_{3}$. Such a line is a bitangent to the curve $q_{i}=0$ if we can find further $b, c$ such that the following polynomial vanishes

$$
\begin{array}{ll}
q_{1}\left(a_{2} x_{2}+a_{3} x_{3}, x_{3}, x_{2}\right)-8\left(a_{2}^{4}-1\right)\left(x_{2}^{2}+b x_{2} x_{3}+c x_{3}^{2}\right)^{2} & \text { if } i=1, \\
q_{2}\left(a_{2} x_{2}+a_{3} x_{3}, x_{3}, x_{2}\right)-8 a_{2}^{4}\left(x_{2}^{2}+b x_{2} x_{3}+c x_{3}^{2}\right)^{2} & \text { if } i=2, \\
q_{3}\left(a_{2} x_{2}+a_{3} x_{3}, x_{3}, x_{2}\right)-a_{2}^{4}\left(x_{2}^{2}+b x_{2} x_{3}+c x_{3}^{2}\right)^{2} & \text { if } i=3, \\
q_{6}\left(a_{2} x_{2}+a_{3} x_{3}, x_{3}, x_{2}\right)-8 a_{2}^{4}\left(x_{2}^{2}+b x_{2} x_{3}+c x_{3}^{2}\right)^{2} & \text { if } i=6, \\
q_{7}\left(a_{2} x_{2}+a_{3} x_{3}, x_{3}, x_{2}\right)-a_{2}^{4}\left(x_{2}^{2}+b x_{2} x_{3}+c x_{3}^{2}\right)^{2} & \text { if } i=7 .
\end{array}
$$

The factors $8\left(a_{2}^{4}-1\right), 8 a_{2}^{4}$ and $a_{2}^{4}$ are chosen in order to kill the coefficient of $x_{3}^{4}$ in each of the polynomials. Hence each of the five above polynomials is a polynomial of degree 3 in $x_{3}$. These polynomials can be computed with the help of some computeralgebra package. Unfortunately, the obtained expressions are too long to include them here. From these calculations one deduces that both the coefficient of $x_{3}^{3}$ and of $x_{3}^{2}$ are linear in $b$ and $c$. We can solve for $b$ and $c$ and substitute the result. However, in order to solve for $b$ and $c$ we have to divide by $a_{2}^{4}-1$ if $i=1$ and by $a_{2}$ in the other cases, hence for the moment we have to assume that they are nonzero.

We are then left with two nonzero coefficients. The coefficient of $x_{3}^{0}$ is a cubic in $a_{3}$. Eliminating $a_{3}$ leaves a polynomial of degree either 20 (if $i=1$ ) or 24 (if $i \neq 1$ ) in $a_{2}$, which we list below.

Each of the zeroes yields a possible value for $a_{2}$. One easily checks that each value for $a_{2}$ determines a unique value for $a_{3}$. In this way we find 20 or 24 bitangents. Note that each of the five families admits the automorphism $\left(u, v, x_{2}, x_{3}\right) \mapsto\left(u, v,-x_{2},-x_{3}\right)$. This implies that if $\left(a_{2}, a_{3}\right)$ defines a bitangent then so does $\left(-a_{2},-a_{3}\right)$. Hence the final polynomial in $a_{2}$ is actually a polynomial in $a_{2}^{2}$. Depending on the case there are further automorphisms, which could give further simplifications.

We now list for each case the degree 24 polynomial in $a_{2}$. The case $i=1$ is slightly more involved then the other ones, so we start with the case $i \geq 2$.

For $i=2$ we find that if $a_{2}$ is a zero of

$$
\left(\left(\lambda^{2}+16\right) a_{2}^{4}+4 \lambda a_{2}^{2} \lambda+2\right)\left(\left(\lambda^{2}-16\right) a_{2}^{4}+4 \lambda a_{2}^{2} \lambda+2\right) \cdots
$$




$$
\begin{aligned}
& \cdots\left(1024 a_{2}^{16}+128 \lambda^{3} a_{2}^{14}+\left(2 \lambda^{6}+960 \lambda^{2}\right) a_{2}^{12}+\left(20 \lambda^{5}+2560\right) a_{2}^{10}+\cdots\right. \\
& \left.\cdots+\left(73 \lambda^{4}+2176\right) a_{2}^{8}+120 \lambda^{3} a_{2}^{6}+92 \lambda^{2} a_{2}^{4}+32 \lambda a_{2}^{2}+4\right)
\end{aligned}
$$

then there is a unique $a_{3}$ yielding a bitangent. The degree 16 factor can be written as the product of two factors of degree 8 over $\mathbf{F}_{q}(\sqrt{2})$. This yields 24 of the 28 bitangents.

For $i=3$ we find that $a_{2}$ is a zero of

$$
\begin{gathered}
\left(2 a_{2}^{4}+t a_{2}^{2}+2\right)\left(2 a_{2}^{4}-t a_{2}^{2}-2\right) \cdots\left(a_{2}^{8}+4 a_{2}^{6}+\left(\lambda^{2}-4 \lambda+8\right) a_{2}^{4}+(4 \lambda-8) a_{2}^{2}+4\right) \cdots \\
\cdots\left(a_{2}^{8}-4 a_{2}^{6}+\left(\lambda^{2}+4 \lambda+8\right) a_{2}^{4}+(4 \lambda+8) a_{2}^{2}+4\right)
\end{gathered}
$$

then there is a unique $a_{3}$ yielding a bitangent. Each of the two degree 8 factors is a product of two factors of degree 4 factors over $\mathbf{F}_{q}(\sqrt{-1})$. This yields 24 of the 28 bitangents.

For $i=4$ we find that $a_{2}$ is a zero of

$$
\begin{aligned}
& \left(-512 \lambda^{6}-1769472\right) a_{2}^{24}-9216 \lambda^{5} a_{2}^{22}+\left(2 \lambda^{10}-62976 \lambda^{4}\right) a_{2}^{20}+\cdots \\
& \quad \cdots\left(36 \lambda^{9}-286720 \lambda^{3}\right) a_{2}^{18}+\left(273 \lambda^{8}-663552 \lambda^{2}\right) a_{2}^{16}+\cdots \\
& \quad \cdots+\left(1136 \lambda^{7}-700416 \lambda\right) a_{2}^{14}+\left(2840 \lambda^{6}-276480\right) a_{2}^{12}+4416 \lambda^{5} a_{2}^{10}+\cdots \\
& \quad \cdots+4312 \lambda^{4} a_{2}^{8}+2624 \lambda^{3} a_{2}^{6}+960 \lambda^{2} a_{2}^{4}+192 \lambda a_{2}^{2}+16
\end{aligned}
$$

then there is a unique $a_{3}$ yielding a bitangent. This yields 24 of the 28 bitangents.

For $i=5$ we find that $a_{2}$ is a zero of

$$
\begin{aligned}
& \left(-3 a_{2}^{8}+2 \lambda a_{2}^{6}+\lambda^{2} a_{2}^{4}+12 a_{2}^{4}+4 \lambda a_{2}^{2}+4\right) \cdots \\
& \quad \cdots\left(9 a_{2}^{16}+6 \lambda a_{2}^{14}+\left(7 \lambda^{2} a_{2}^{12}+36\right) a_{2}^{12}+\left(60-2 \lambda^{3}\right) a_{2}^{10} \lambda+\cdots\right. \\
& \left.\quad \cdots+\left(\lambda^{4}-20 \lambda^{2}+156\right) a_{2}^{8}+\left(8 \lambda^{3}-56 \lambda\right) a_{2}^{6}+\left(24 \lambda^{2}-48\right) a_{2}^{4}+32 a_{2}^{2} \lambda+16\right)
\end{aligned}
$$

then there is a unique $a_{3}$ yielding a bitangent. This yields 24 of the 28 bitangents. If $\omega^{2}=-\omega-1$ then over $\mathbf{F}_{q}(\omega)$ we can write the degree 16 factor as a product of two factors of degree 8 .

To finish the cases $i=2,3,6,7$ we need to find 4 further bitangents. The above approach gives all bitangents of the form $u=a_{2} x_{2}+a_{3} x_{3}$ with $a_{2} \neq 0$. It turns out that there are no bitangents with $a_{2}=0$, however there are bitangents of the form $a_{2} x_{2}+a_{3} x_{2}=0$. One easily sees that the line $x_{3}=0$ is a hyperflex line (and therefore a bitangent) and that the remaining three bitangents are of the form $x_{2}=a x_{3}$, with $a$ a zero of

$$
\begin{array}{ll}
a\left(8 a^{2}+\lambda^{2} a+8\right) & \text { if } \quad i=2, \\
a\left(a^{2}+1\right) & \text { if } \quad i=3, \\
8 a^{3}+\lambda^{2} a^{2}+2 & \text { if } \quad i=6, \\
(a+1)\left(a^{2}-a+1\right) & \text { if } \quad i=7 .
\end{array}
$$

In the cases $i=2,3,6,7$ we find that 24 of the bitangents can be described in terms of a polynomial in $a_{2}$ of degree 24. Since $a_{1}$ is the unique root of a polynomial with coefficients in $\mathbf{F}_{q}\left(\lambda, a_{2}\right)$ we find that $a_{1} \in \mathbf{F}_{q}\left(\lambda, a_{2}\right)$. The equations defining $c$ and $d$ are linear, hence they are also in $\mathbf{F}_{q}\left(\lambda, a_{2}\right)$. Hence the bitangent is defined over $\mathbf{F}_{q}\left(\lambda, a_{2}\right)$. However, the lines on the del Pezzo surface may be defined over a degree 2 extension. If $\ell=V\left(-u+a x_{2}+b x_{3}\right)$ then the equation for the del Pezzo surface is a quadratic equation in $v$. It restriction to $u=a_{1} x_{2}+a_{2} x_{3}$ is a quadratic equation with discriminant $\left.q_{i}\right|_{\ell}$. This discriminant is of the form $C_{i}\left(x_{2}^{2}+b_{i} x_{2} x_{3}+c_{i} x_{3}^{2}\right)^{2}$. Hence to define each of the two corresponding lines on $S_{\lambda}^{(1, i)}$ we need to take a square root of $C$. An explicit calculation now show that $C$ depends only on $i$ and $a_{2}$. More precisely, we have that $C_{i}$ equals $8 a_{2}^{4}, a_{2}^{4}, 8 a_{2}^{4}, a_{2}^{4}$ for $i=2,3,6,7$. Hence for $i=3,7$ both lines are defined over $\mathbf{F}_{q}\left(\lambda, a_{2}\right)$ but for $i=2,6$ they are defined over $\mathbf{F}_{q}\left(\lambda, a_{2}, \sqrt{2}\right)$. 
Similarly, one easily checks that the flex line is defined over $\mathbf{F}_{q}$ and that the two corresponding lines on the del Pezzo surface are defined over $\mathbf{F}_{q}$ if $i=3,7$ and over $\mathbf{F}_{q}(\sqrt{-1})$ if $i=2,6$ and that each of the remaining lines are defined over $\mathbf{F}_{q}(a)$ if $i=3,7$ and $\mathbf{F}_{q}(\sqrt{2}, \lambda, a)$ if $i=2,6$.

For $i=1$ we can copy the above approach, but in the first step we find 20 rather than 24 bitangents of the form $u=a_{2} x_{2}+a_{3} x_{3}$. These 20 bitangents are one of the following (where $I$ is a fixed root of -1 ):

1) $a_{3}=a_{2}$ and $a_{2}^{4}\left(\lambda^{2}+16\right)-16 \lambda a_{2}^{2}+\lambda^{2}+16=0$,

2) $a_{3}=-a_{2}$ and $a_{2}^{4}\left(\lambda^{2}+16\right)+16 \lambda a_{2}^{2}+\lambda^{2}+16=0$,

3) $a_{3}=I a_{2}$ and $a_{2}^{4}\left(\lambda^{2}-16\right)-16 I \lambda a_{2}^{2}+\lambda^{2}-16=0$,

4) $a_{3}=-I a_{2}$ and $a_{2}^{4}\left(\lambda^{2}-16\right)-16 I \lambda a_{2}^{2}+\lambda^{2}-16=0$,

5) $a_{3}=\frac{t}{4 a_{2}}$ and $a_{3}^{4}=1$.

Using symmetry we find that further bitangents are given by $a_{3}=\frac{t}{4 a_{2}}$ and $a_{2}^{4}=1$.

There are four further bitangents of the form $x_{2}=a x_{3}$ with

$$
8 a^{4}+\lambda^{2} a^{2}+8=0 .
$$

One easily checks that the corresponding lines on the del Pezzo surface are defined over the field $\mathbf{F}_{q}\left(\lambda, a_{2}, \sqrt{2\left(a_{2}^{4}-1\right)}\right)$ (if $\left.a_{2}^{4} \neq 1\right)$, over $\mathbf{F}_{q}\left(\lambda, a_{1}, \sqrt{2\left(a_{3}^{4}-1\right)}\right)$ (if $a_{3}^{4} \neq 1$ ) and over $\mathbf{F}_{q}(\lambda, a, \sqrt{2})$ (for the final four lines).

\section{A.2 $S_{\lambda}^{(i, 2)}$ and $S_{\lambda}^{(i, 3)}$}

Once we found the lines on $S_{\lambda}^{(i, 1)}$ we can use them to find also the lines on $S_{\lambda}^{(i, 2)}$ and $S_{\lambda}^{(i, 3)}$. Let

$$
h_{3}\left(u, v, x_{2}, x_{3}\right):=u^{4}+4 u^{2} v+2 v^{2}+\lambda v x_{2} x_{3}, \quad h_{4}\left(u, v, x_{2}, x_{3}\right):=v\left(u^{2}+2 v\right)+\lambda v x_{2} x_{3} .
$$

Proceeding as above we find that that $S_{\lambda}^{(i, 2)}$ is defined by

$$
h_{3}+g_{1}, \quad h_{4}+g_{2}, \quad h_{4}+g_{2}, \quad h_{3}+g_{3}, \quad h_{4}+g_{3} .
$$

The map $\left(u, v, x_{2}, x_{3}\right) \mapsto\left(u I, v, x_{2}, x_{3}\right)$ defines an isomorphism $S_{\lambda}^{(i, 2)} \rightarrow S_{\lambda}^{(i, 1)}$ for $i=1,2,6$. The $\operatorname{map}\left(u, v, x_{2}, x_{3}\right) \mapsto\left(u I,-v, I x_{2}, I x_{3}\right)$ defines an isomorphism $S_{\lambda}^{(i, 2)} \rightarrow S_{\lambda}^{(i, 1)}$ for $i=3,7$.

Let $h_{5}\left(u, v, x_{2}, x_{3}\right):=u^{4}-4 I u^{2} v-2 v^{2}+\lambda v x_{2} x_{3}$ then $S_{\lambda}^{(i, 3)}$ is defined (for $i=1,2,6$ ) by

$$
h_{5}+g_{1}, \quad h_{5}+g_{2}, \quad h_{5}+g_{3} .
$$

The map $\left(u, v, x_{2}, x_{3}\right) \mapsto\left(u, I v, x_{2}, I x_{3}\right)$ defines an isomorphism $S_{\lambda}^{(i, 3)} \rightarrow S_{\lambda}^{(i, 2)}$ for $i=1$. The $\operatorname{map}\left(u, v, x_{2}, x_{3}\right) \mapsto\left(u, I v, \zeta x_{2}, \zeta^{5} x_{3}\right)$ defines an isomorphism $S_{\lambda}^{(i, 3)} \rightarrow S_{\lambda}^{(i, 2)}$ for $i=2$.

For $i=7$ we need also to act on $\lambda$ : The map $\left(u, v, x_{2}, x_{3}\right) \mapsto\left(u, I v, x_{2}, x_{3}\right)$ defines an isomorphism $S_{-I \lambda}^{(i, 3)} \rightarrow S_{\lambda}^{(i, 2)}$ for $i=2$.

Substituting $v=x_{0} x_{1}$ and $u=x_{0}+x_{1}$ (if $j=1$ ), $u=x-y$ (if $j=2$ ) or $u=x+I y$ (if $j=3$ ) in the equations of a line on $S_{\lambda}^{(i, j)}$ then yields the corresponding conic on $X_{\lambda}^{(i, j)}$.

\section{Acknowledgements}

The author would like to thank John Voight and Tyler Kelly for various conversations on this topic. The author would like to thank the referees for various suggestions to improve the exposition. 


\section{References}

[1] Baldassarri F., Chiarellotto B., Algebraic versus rigid cohomology with logarithmic coefficients, in Barsotti Symposium in Algebraic Geometry (Abano Terme, 1991), Perspect. Math., Vol. 15, Academic Press, San Diego, CA, 1994, 11-50.

[2] Berthelot P., Géométrie rigide et cohomologie des variétés algébriques de caractéristique $p$, in Study Group on Ultrametric Analysis, 9th year: 1981/82, No. 3 (Marseille, 1982), Inst. Henri Poincaré, Paris, 1983, Exp. No. J2, 18 pages.

[3] Berthelot P., Dualité de Poincaré et formule de Künneth en cohomologie rigide, C. R. Acad. Sci. Paris Sér. I Math. 325 (1997), 493-498.

[4] Bini G., Quotients of hypersurfaces in weighted projective space, Adv. Geom. 11 (2011), 653-667, arXiv:0905.2099.

[5] Bini G., van Geemen B., Kelly T.L., Mirror quintics, discrete symmetries and Shioda maps, J. Algebraic Geom. 21 (2012), 401-412, arXiv:0809.1791.

[6] Candelas P., de la Ossa X., Rodriguez-Villegas F., Calabi-Yau manifolds over finite fields. II, in Calabi-Yau Varieties and Mirror Symmetry (Toronto, ON, 2001), Fields Inst. Commun., Vol. 38, Amer. Math. Soc., Providence, RI, 2003, 121-157, hep-th/0402133.

[7] Chahal J., Meijer M., Top J., Sections on certain $j=0$ elliptic surfaces, Comment. Math. Univ. St. Paul. 49 (2000), 79-89, math.NT/9911274.

[8] Doran C.F., Kelly T.L., Salerno A., Sperber S., Voight J., Whitcher U., Zeta functions of alternate mirror Calabi-Yau families, arXiv:1612.09249.

[9] Dwork B., p-adic cycles, Inst. Hautes Études Sci. Publ. Math. (1969), 27-115.

[10] Gährs S., Picard-Fuchs equations of special one-parameter families of invertible polynomials, Ph.D. Thesis, der Gottfried Wilhelm Leibniz Universität Hannover, 2011, arXiv:1109.3462.

[11] Griffiths P.A., On the periods of certain rational integrals. II, Ann. of Math. 90 (1969), 496-541.

[12] Katz N.M., On the differential equations satisfied by period matrices, Inst. Hautes Études Sci. Publ. Math. (1968), 223-258.

[13] Kelly T.L., Berglund-Hübsch-Krawitz mirrors via Shioda maps, Adv. Theor. Math. Phys. 17 (2013), 14251449, arXiv:1304.3417.

[14] Kloosterman R., Explicit sections on Kuwata's elliptic surfaces, Comment. Math. Univ. St. Pauli 54 (2005), 69-86, math.AG/0502017.

[15] Kloosterman R., The zeta function of monomial deformations of Fermat hypersurfaces, Algebra Number Theory 1 (2007), 421-450, math.NT/0703120.

[16] Kloosterman R., Group actions on rigid cohomology with compact support - Erratum to "The zeta function of monomial deformations of Fermat hypersurfaces", Preprint.

[17] Lauder A.G.B., Deformation theory and the computation of zeta functions, Proc. London Math. Soc. 88 (2004), 565-602.

[18] Pancratz S., Tuitman J., Improvements to the deformation method for counting points on smooth projective hypersurfaces, Found. Comput. Math. 15 (2015), 1413-1464, arXiv:1307.1250.

[19] Scholten J., Mordell-Weil groups of elliptic surfaces and Galois representations, Ph.D. Thesis, Rijksuniversiteit Groningen, 2000.

[20] Shioda T., An explicit algorithm for computing the Picard number of certain algebraic surfaces, Amer. J. Math. 108 (1986), 415-432.

[21] van der Put M., The cohomology of Monsky and Washnitzer, Mém. Soc. Math. France (N.S.) (1986), 33-59. 